\title{
Review of Femtosecond-Laser-Inscribed Fiber Bragg Gratings: Fabrication Technologies and Sensing Applications
}

\author{
Jun $\mathrm{HE}^{1,2}$, Baijie $\mathrm{XU}^{1,2}$, Xizhen $\mathrm{XU}^{1,2}$, Changrui LIAO ${ }^{1,2}$, and Yiping $\mathrm{WANG}^{1,2^{*}}$ \\ ${ }^{1}$ Key Laboratory of Optoelectronic Devices and Systems of Ministry of Education/Guangdong Province, College of \\ Physics and Optoelectronic Engineering, Shenzhen University, Shenzhen 518060, China \\ ${ }^{2}$ Shenzhen Key Laboratory of Photonic Devices and Sensing Systems for Internet of Things, Guangdong and Hong Kong \\ Joint Research Centre for Optical Fibre Sensors, Shenzhen University, Shenzhen 518060, China \\ ${ }^{*}$ Corresponding author: Yiping WANG_ E-mail: ypwang@szu.edu.cn
}

\begin{abstract}
Fiber Bragg grating (FBG) is the most widely used optical fiber sensor due to its compact size, high sensitivity, and easiness for multiplexing. Conventional FBGs fabricated by using an ultraviolet (UV) laser phase-mask method require the sensitization of the optical fiber and could not be used at high temperatures. Recently, the fabrication of FBGs by using a femtosecond laser has attracted extensive interests due to its excellent flexibility in creating FBGs array or special FBGs with complex spectra. The femtosecond laser could also be used for inscribing various FBGs on almost all fiber types, even fibers without any photosensitivity. Such femtosecond-laser-induced FBGs exhibit excellent thermal stability, which is suitable for sensing in harsh environment. In this review, we present the historical developments and recent advances in the fabrication technologies and sensing applications of femtosecond-laser-inscribed FBGs. Firstly, the mechanism of femtosecond-laser-induced material modification is introduced. And then, three different fabrication technologies, i.e., femtosecond laser phase mask technology, femtosecond laser holographic interferometry, and femtosecond laser direct writing technology, are discussed. Finally, the advances in high-temperature sensing applications and vector bending sensing applications of various femtosecond-laser-inscribed FBGs are summarized. Such femtosecond-laser-inscribed FBGs are promising in many industrial areas, such as aerospace vehicles, nuclear plants, oil and gas explorations, and advanced robotics in harsh environments.
\end{abstract}

Keywords: Fiber Bragg grating (FBG); femtosecond laser; high temperature sensor; vector bending sensor

Citation: Jun HE, Baijie XU, Xizhen XU, Changrui LIAO, and Yiping WANG, "Review of Femtosecond-Laser-Inscribed Fiber Bragg Gratings: Fabrication Technologies and Sensing Applications," Photonic Sensors, 2021, 11(2): 203-226.

\section{Introduction}

Fiber Bragg grating (FBG) is a critical optical fiber component used in modern information technologies. An FBG is formed by a permanent periodic refractive index modulation created in the fiber core and exhibits narrow-band Bragg reflection due to the coupling of the forward and backward propagation modes [1]. The research on FBGs began as early as 1970 s, since the first FBG was created by Hill et al. [2] in 1978. In 1989, Meltz et al. [3] first reported the inscription of FBGs by using a novel side-exposure method based on ultraviolet (UV) laser holographic interferometry. In 1993, the researchers at Bell laboratory found that the fiber photosensitivity could be increased by hydrogen

Received: 22 March 2021 / Revised: 27 March 2021

(C) The Author(s) 2021. This article is published with open access at Springerlink.com

DOI: $10.1007 / \mathrm{s} 13320-021-0629-2$

Article type: Review 
loading [4]. In the same year, Hill et al. [5] invented another FBG fabrication technology based on UV laser through a phase mask. This technology is quite simple, stable, and efficient for mass production of FBGs, and hence achieves commercial success in the following decades. Owing to the development of UV laser phase mask technology, various FBGs were successfully fabricated and widely used as filters or sensors in many industrial areas, such as fiber-optic communication systems, optical fiber sensor networks, fiber lasers, and microwave photonics [6-9]. Moreover, FBGs operating at high temperatures are critical in applications such as sensors in harsh environments. Unfortunately, conventional FBGs with type I index modulation created by using UV laser phase mask technology are well suited for operating temperatures below $450^{\circ} \mathrm{C}$ [8]. However, sensing in harsh environments, such as the oil and gas industries, power stations, aircraft engines, and furnaces, requires high operating temperatures from $400{ }^{\circ} \mathrm{C}$ to $1800{ }^{\circ} \mathrm{C}$. In addition, conventional FBGs inscribed in telecommunication single-mode fibers (SMFs) are typically insensitive to vector bending and could not be used in many applications such as medical instruments and robotics.

Researchers have been exploring new technologies for fabricating novel FBGs that could operate at high temperatures or used as vector bending sensors in harsh environments. Femtosecond laser is a powerful tool for fabricating various microstructures inside transparent materials [10-15]. Due to its very short pulse width and extremely high peak power, the femtosecond laser can induce effective refractive index change in almost all kinds of transparent materials, such as single crystals, glasses, and optical fibers. In 2001, Oi et al. [16] first reported the inscription of FBGs by using near infrared (NIR) femtosecond laser together with a holographic interferometer. In 2003, Dragomir et al. [17] and Mihailov et al. [18] reported separately the fabrications of FBGs by using a UV femtosecond laser phase mask method and a NIR femtosecond laser phase mask method. In 2004, Martinez et al. [19] demonstrated the fabrication of FBGs by using a femtosecond laser point-by-point technology. Since then, the mechanisms, fabrication technologies, and sensing applications of femtosecond-laser-induced FBGs have been developed rapidly. Owing to the continuous progress in fabrication technologies, high-quality FBGs, featuring by high reflectivity, low insertion loss, low polarization-dependent loss (PDL), and low cladding mode loss, have been fabricated successfully by using femtosecond laser [20-23]. Special FBGs with complex spectra, such as apodized FBGs, sampled FBGs, chirped FBGs, and phase-shifted FBGs, have also been created by femtosecond laser inscription [24-29]. Moreover, FBGs have also been inscribed successfully in various specialty optical fibers, such as pure silica core SMF [30], side hole micro-structured fiber [31], multicore fibers (MCFs) [32, 33], photonic crystal fibers (PCFs) [34-37], rare-earth-doped silica fibers [38-40], fluoride fibers (ZBLAN) [41-45], and single-crystal sapphire fibers [46-49].

Subsequently, researchers began to exploit the applications of femtosecond-laser-inscribed FBGs in fiber sensors and lasers [50]. For example, high-quality wavelength-specific FBGs were directly inscribed in non-photosensitive active fibers by using femtosecond laser, resulting in a novel ytterbium fiber laser operating at $\sim 1073 \mathrm{~nm}$ [51], erbium fiber laser operating at $\mathrm{C}$ band $(\sim 1554.5 \mathrm{~nm})$ [52], and fluoride fiber lasers operating at mid-infrared (MIR) region $(\sim 2.8 \mu \mathrm{m})$ [53, 54]. Moreover, the femtosecond-laser-inscribed FBGs were also used as high-temperature sensors. For instance, a type II FBG fabricated on conventional SMF exhibited an excellent long-term thermal stability at $1000^{\circ} \mathrm{C}$ for several hundred hours [55]. An FBG fabricated on pure-silica PCF could operate at $\sim 1300{ }^{\circ} \mathrm{C} \quad$ [56]. An $\mathrm{FBG}$ fabricated on single-crystal sapphire fiber can operate at a very high temperature of up to $1900{ }^{\circ} \mathrm{C}$ in tube furnace [57]. Additionally, shape sensing in harsh 
environment could also be realized based on FBGs inscribed on MCFs by using a femtosecond laser [58].

In this paper, we review the historical developments and recent advances in the fabrication technologies and sensing applications of femtosecond-laser-inscribed FBGs. In Section 1, we introduce the mechanism of femtosecond-laserinduced material modification and three different fabrication technologies, i.e. femtosecond laser phase mask technology, femtosecond laser holographic interferometry, and femtosecond laser direct writing technology. In Sections 2 and 3, we introduce the advances in the applications of femtosecond-laser-inscribed FBGs for hightemperature sensing and vector bending sensing, respectively. In particular, we introduce the recent progress at Shenzhen University, China. In the last section, we summarize the progress and challenges in the fabrication technologies and sensing applications of femtosecond-laser-inscribed FBGs.

\section{Femtosecond laser inscription of FBGs}

Femtosecond laser pulses can induce effective refractive index modulation in almost all types of optical materials, including non-photosensitive optical fibers. To date, three mainstream fabrication technologies, i.e., femtosecond laser phase mask technology, femtosecond laser holographic interferometry, and femtosecond laser direct writing technology, have been developed for fabricating FBGs in various fiber types.

\subsection{Mechanism of material modification induced by femtosecond laser}

Femtosecond laser micromachining has attracted tremendous attention in industries due to its advantages of high quality, high precision, and internal processing capability. The femtosecond laser pulses, featuring by ultra-short pulse width and extremely-high peak intensity, can induce significant refractive index modulation in different types of materials [10-12]. As shown in Fig. 1, when a femtosecond laser beam is focused inside transparent material through a high numerical aperture (NA) objective lens, the high laser intensity can initiate nonlinear absorption within the focal volume through field ionization and avalanche ionization, as shown in Fig. 1(b). Consequently, a large amount of electron-ion plasmas are created in the focal volume, leading to the permanent refractive index modulation through the process of plasmas combination and energy dissipation. Three different types of refractive index modulation, i.e., isotropic index increase, anisotropic index change (birefringence), and voids, could be created in the transparent material depending on the pulse energy used at a specific pulse width of the femtosecond laser, as shown in Fig. 1(c) [11].

(1) In the case of low-energy femtosecond laser pulses, melting and subsequent rapid solidification will occur in the focal volume, resulting in an isotropic refractive index increase in the sample. Hence, low-energy femtosecond laser pulses are widely used for inscribing type I optical waveguides [13] or FBGs [59, 60] inside the optical fiber.

(2) In the case of intermediate-energy femtosecond laser pulses, nanogratings, i.e., periodic nanometer-scale stripes perpendicular to the incident polarization of the femtosecond laser beam, will be generated in the focal volume, resulting in an anisotropic refractive index change (i.e., birefringence) in the sample. These nanogratings could be used for fabricating novel polarization devices [61, 62].

(3) In the case of high-energy femtosecond laser pulses, micro-explosion will occur in the focal volume. The material expands explosively out of the vocal volume and into the surrounding material and hence results in a void in the sample. Therefore, high-energy femtosecond laser pulses are widely used for fabricating micro-holes, micro-cavities, or micro-channels inside transparent materials [15, 63], and also can be used for fabricating type II FBGs with enhanced thermal stability $[60,64]$. 
(a)

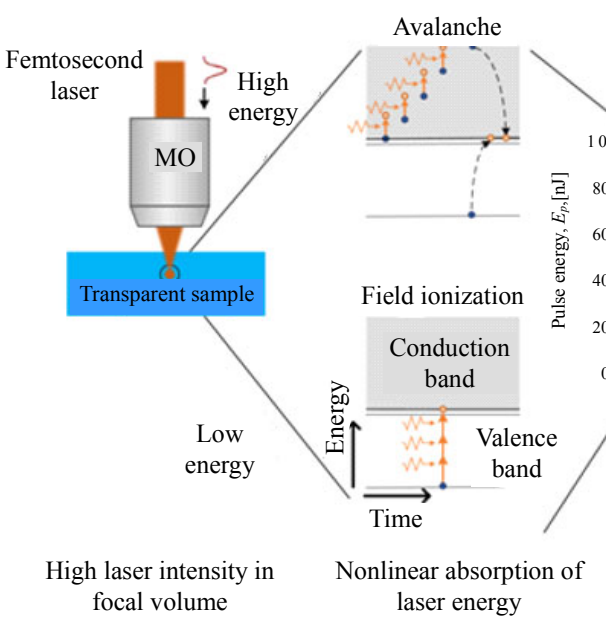

(c)

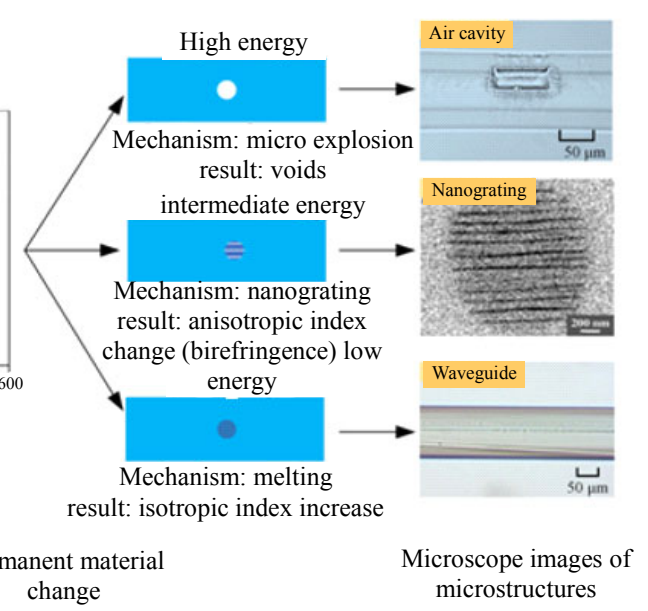

Fig. 1 Schematic diagram of the mechanism [key steps: (a), (b), and c] and typical results (d) of three different types of refractive index modulation induced by femtosecond laser (Note that part of the insets were taken from [11, 13-15]).

\subsection{Femtosecond laser phase mask technology}

The femtosecond laser phase mask technology was proposed and first demonstrated for fabricating FBGs as early as in 2003 by Mihailov et al. [18] at Communications Research Centre Canada (CRC). The setup for fabricating FBGs by using this technology is similar to that of a conventional UV laser phase mask technology, except for the deployment of a femtosecond laser for side exposure on the optical fiber. As shown in Fig.2(a), the laser beam is focused onto the fiber core by using a cylindrical lens through a uniform silica phase mask. The fiber is placed inside the interference area of the overlapped \pm 1 order diffraction of the phase mask, and hence an FBG can be inscribed in the fiber core. At early stages, it was difficult to obtain a first-order phase mask with adequate suppression of 0 -order diffraction. In order to avoid the multiple beam interference generated in fiber cladding, Smelser et al. $[65,66]$ used a walk-off effect to generate pure two-beam interference at appropriate fiber-mask distances, and hence Grobnic et al. [20] fabricated FBGs with extremely low cladding mode resonance. Moreover, they found that the hydrogen loading can effectively reduce the thresholds for fabricating FBGs by using the femtosecond laser phase mask technology [59]. In addition, FBGs could also be inscribed onto the fiber core through the polymer coating by using femtosecond laser phase mask technology with tightly-focusing cylindrical lenses [67-69]. For example, Bernier et al. [68] at Laval University fabricated a highly reflective first-order FBG through the polyimide coating of pure silica core fiber, and this FBG exhibited very high mechanical strength. In 2017, Mihailov et al. [69] successfully fabricated an ultra-short FBG (on the order of $100 \mu \mathrm{m}$ in length) through the fiber coating by use of this technology with a slit placed proximate the mask.

At Shenzhen University, we also studied the FBG inscription by using the femtosecond laser phase mask technology. A Ti:sapphire regenerative amplified femtosecond laser (Spectra-Physics, Solstice 100PF) with a wavelength of $800 \mathrm{~nm}$, a pulse-width of $100 \mathrm{fs}$, and a repetition rate of $1 \mathrm{kHz}$, a cylindrical lens with a focal length of $50.2 \mathrm{~mm}$, and a high-quality uniform first-order phase mask (Ibsen Photonics) with a period of $1070 \mathrm{~nm}$, a 0th order diffraction of below $4 \%$, and optimized for $800 \mathrm{~nm}$ TE illumination, were used in our setup. The fiber was placed at a distance of $\sim 300 \mu \mathrm{m}$ behind the phase mask. Using Gaussian beam optics, the 


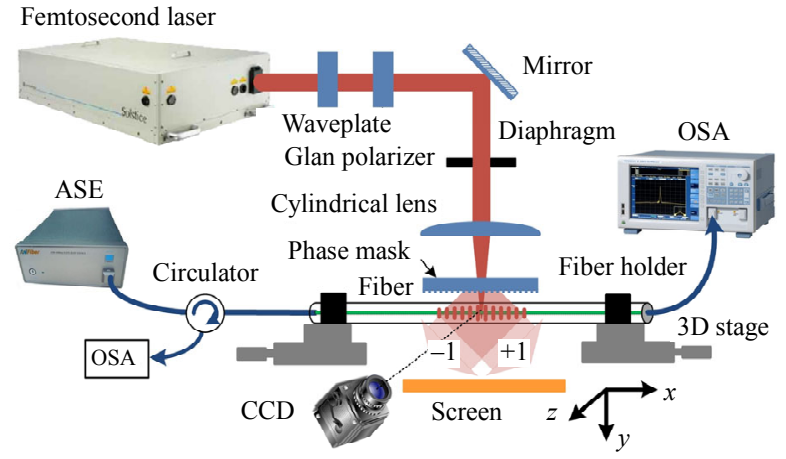

(a)

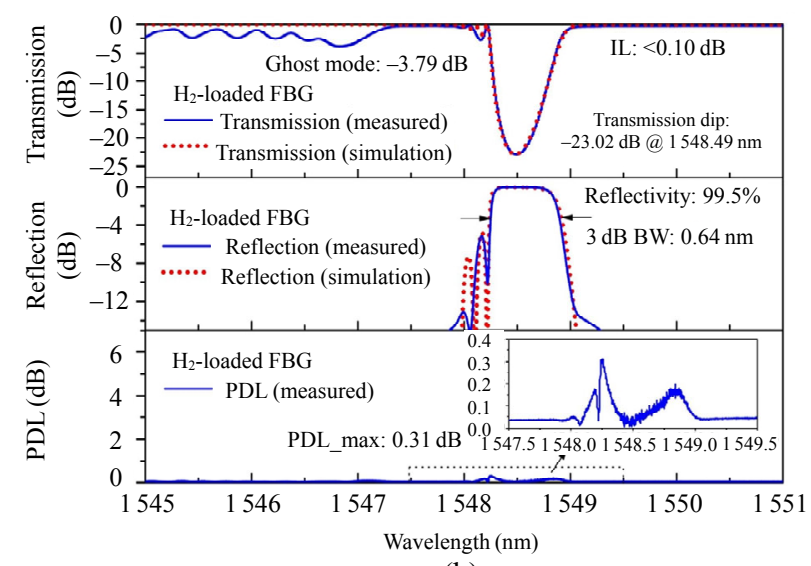

(b)

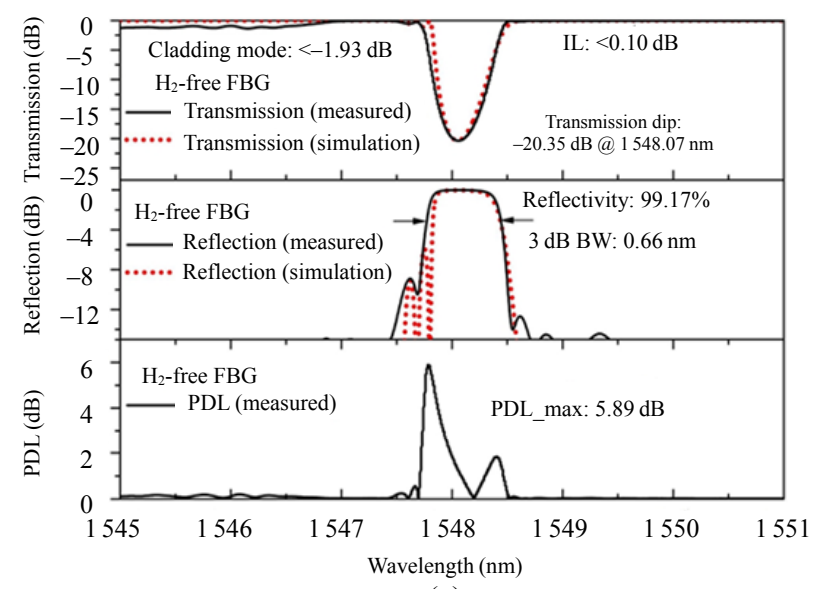

(c)

Fig. 2 Femtosecond laser phase mask technology for fabricating FBGs: (a) schematics of the experimental setup, and measured and calculated results of two fabricated FBGs in (b) $\mathrm{H}_{2}$-loaded fiber and (c) $\mathrm{H}_{2}$-free fiber, respectively.

focal width and Rayleigh length of the laser beam are calculated to be $8.25 \mu \mathrm{m}$ and $66.78 \mu \mathrm{m}$, respectively, which are compatible to the fiber core diameters and efficient for FBG inscription. Therefore, we successfully fabricated high-quality FBGs in both $\mathrm{H}_{2}$-loaded and $\mathrm{H}_{2}$-free fibers by using this technology. As shown in Figs.2(b) and 2(c), the measured spectra of the fabricated FBGs agree well with the theoretical calculations. Moreover, both the $\mathrm{H}_{2}$-loaded FBG and $\mathrm{H}_{2}$-free $\mathrm{FBG}$ exhibit high reflectivity, low insertion loss (IL), and low cladding mode loss, whereas the $\mathrm{H}_{2}$-loaded FBG also exhibits a very low PDL of below $0.3 \mathrm{~dB}$, resulting from the very low pulse energy of $\sim 48 \mu \mathrm{J}$ used for inscribing such a $\mathrm{H}_{2}$-loaded FBG.

The grating pitch of an FBG fabricated by using the femtosecond laser phase mask technology is exactly one half of the phase mask period, such as phase-shifted FBGs or chirped FBGs (CFBGs). Therefore, various methods have been proposed to overcome this limitation. For example, in 2015, we reported a novel highly birefringent phase-shifted FBG inscribed in SMF with a uniform phase mask and femtosecond laser overexposure [62]. In the same year, Bernier et al. [53] reported a 30 -mm-long $\pi$-phase-shifted FBG inscribed in a heavily erbium-doped fluoride fiber and achieved an all-fiber distributed feedback (DFB) laser operating at $2.8 \mu \mathrm{m}$. In 2019, Mihailov et al. [70] reported the fabrication of novel $\pi$-phase-shifted FBGs and FBGs-based Fabry-Perot structures by using a shielded femtosecond laser phase mask technology. Moreover, various CFBGs have also been demonstrated. For example, as shown in Fig. 3(a), Thomas et al. [71] reported a new CFBG fabrication method by bending the fiber under a uniform phase mask. The fabricated CFBG exhibits a bandwidth of $6 \mathrm{~nm}$. Later, they proposed another CFBG fabrication method based on a poly (methyl-methacrylate) (PMMA) phase mask [72]. As shown in Fig. 3(b), the grating pitch of the fabricated FBG can be tuned during the writing process by heating the PMMA phase mask, which has high thermal expansion coefficient. In addition, an ultra-broadband FBG, with a high reflectivity across the five telecom bands [as shown in Fig.3(c), FHWM: $310 \mathrm{~nm}, \mathrm{E}+\mathrm{S}+\mathrm{C}+\mathrm{L}+\mathrm{U}]$, was successfully fabricated by Bernier et al. [73] using NIR femtosecond pulses through a highly chirped first-order phase-mask. 

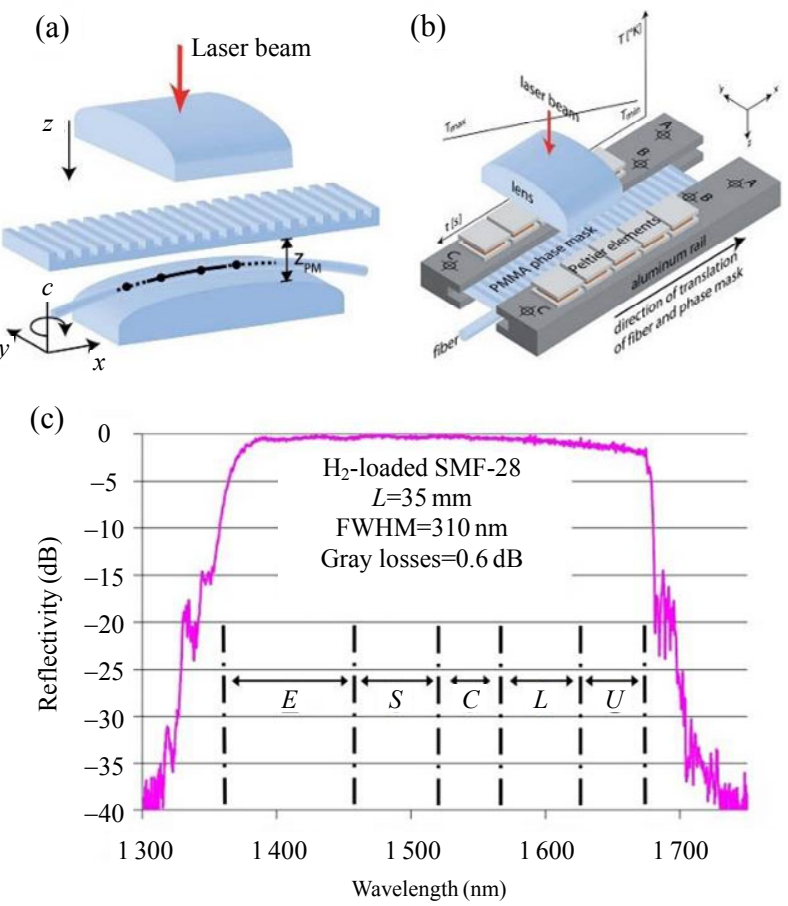

Fig. 3 Fabrication of chirped FBGs by using femtosecond laser phase mask technology: (a) bending the fiber under a uniform phase mask [71], (b) using a tunable PMMA phase mask [72], and (c) measured reflection spectrum of an ultra-broadband CFBG inscribed with a highly chirped first-order phase-mask [73].

Furthermore, FBGs inscribed in fiber cladding are valuable for fiber sensors and fiber lasers [74]. These cladding FBGs typically have strong cladding mode resonances in transmission spectra. For instance, Rong et al. [75] reported a directional fiber-optic accelerometer based on a femtosecond-laser-inscribed cladding FBG in a thin-core fiber. In 2019, Talbot et al. [76] inscribed a CFBG in the pure silica inner cladding of erbium-doped gain fiber using a $400 \mathrm{~nm}$ femtosecond laser phase mask technology. The cladding FBG functioned as a pump reflector and could increase the slope efficiency of a cladding-pumped $25 \mathrm{~W}$ all-fiber laser operating at $1.6 \mu \mathrm{m}$. In 2020, Abdukerim et al. [77] observed ultra-strong cladding mode resonance of larger than $40 \mathrm{~dB}$ in the transmission spectrum of an FBG inscribed inside the fiber core by using a tightly-focused femtosecond laser phase mask technology. In addition, this FBG can withstand a high temperature up to $1000{ }^{\circ} \mathrm{C}$.

\subsection{Femtosecond laser holographic interferometry}

The femtosecond laser phase mask technology can be used for fabricating high-quality FBGs on various fiber types. However, it is difficult to use one phase mask to fabricate FBGs with different Bragg wavelengths. Therefore, femtosecond laser holographic interferometry was proposed and developed for fabricating FBGs, in particular, for fabricating wavelength-division-multiplexing (WDM) FBGs arrays. The setup of fabricating FBGs by using this technology is shown in Fig. 4(a), in which a Talbot interferometer is used consisting of a phase mask for dividing the incident femtosecond laser beam into two beams at \pm 1 order diffractions, a pair of rotation mirrors used for combining the two coherent beams at a pre-defined included angle $2 \beta$, and a fiber placed in the interference pattern. The period of the grating $\Lambda$ is given by

$$
\Lambda=\frac{\lambda_{L}}{2 \sin \beta}
$$

where $\lambda_{L}$ is the wavelength of the laser. According to the Bragg condition $\lambda_{B}=2 n_{\text {eff }} \Lambda$, the Bragg wavelength of the fabricated FBG $\lambda_{B}$ can be represented as

$$
\lambda_{B}=\frac{n_{\mathrm{eff}} \lambda_{L}}{\sin \beta}
$$

where $n_{\text {eff }}$ is the effective refractive index of the fiber core. The included angle of the two coherent beams $2 \beta$ could be precisely changed by fine-tuning the two rotation mirrors, resulting in FBGs with different periods and Bragg wavelengths. Therefore, WDM FBGs arrays could be fabricated by using the femtosecond laser holographic interferometry.

In 2001, Oi et al. [16] first reported the inscription of FBGs by using an NIR femtosecond laser holographic interferometry. And later, in 2008, Becker et al. [78] at the Institute of Photonic Technology, Jena (IPHT) demonstrated the fabrication of FBGs by using a $262 \mathrm{~nm}$ deep-UV femtosecond laser Talbot interferometry. They 
successfully inscribed an FBG array consisting of seven gratings, in which the grating strength is constant over the full wavelength range. Then, they inscribed highly-reflective FBGs (reflectivity: > $90 \%$ ) in rare-earth-doped fibers by using this technology and used them for fiber lasers [79-81]. In addition, they also succeed in fabricating FBGs in non-photosensitive fibers, such as PCFs [82, 83] and sapphire fiber [47]. For example, Wang et al. [82] reported the inscription of FBGs in pure-silica or Ge-doped PCFs, and Elsmann et al. [47] reported the inscription of three WDM FBGs on a single-crystal sapphire optical fiber by using a $400 \mathrm{~nm}$ femtosecond laser holographic interferometry. Furthermore, in 2014, Saliminia et al. [84] at Laval University realized a Bragg wavelength tuning over the conventional telecom C-band using a $266 \mathrm{~nm}$ femtosecond laser holographic interferometry. Figure 4(b) shows the reflection spectrum of the fabricated WDM FBG array consisting of six gratings located in the wavelength range from $1525 \mathrm{~nm}$ to $1565 \mathrm{~nm}$. Recently, we established a Talbot interferometer setup for fabricating novel FBG arrays at Shenzhen University $[85,86]$. We achieved an FBG array with a dense central wavelength separation of $\sim 0.4 \mathrm{~nm}$ over a wide wavelength band (1 $200 \mathrm{~nm}-1900 \mathrm{~nm})$. Hence, more than 1750 FBGs can be serially integrated in theory [85]. Moreover, we also achieved the inscription of FBG arrays parallel-integrated in a seven-core fiber by using a modified Talbot interferometer and a lens scanning method [86]. In general, the femtosecond laser phase mask technology enables an efficient and flexible inscription of FBGs with different Bragg wavelengths. However, it requires high precision, high stability, and high repeatability to establish such a Talbot interferometer, in particular for femtosecond laser, which has a very short coherence length of tens of micrometers [78].

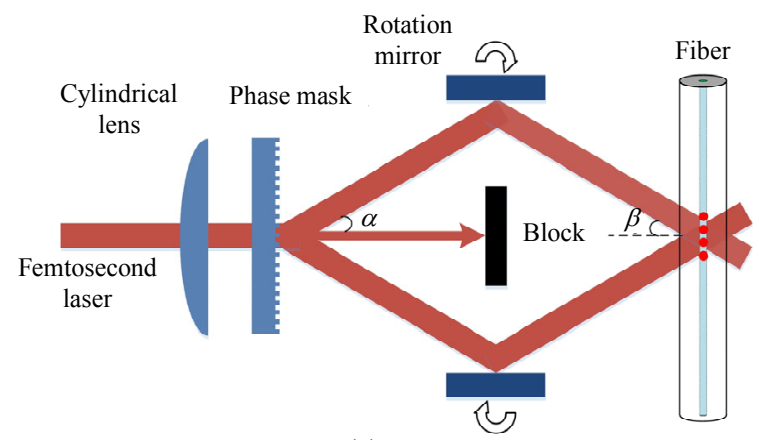

(a)

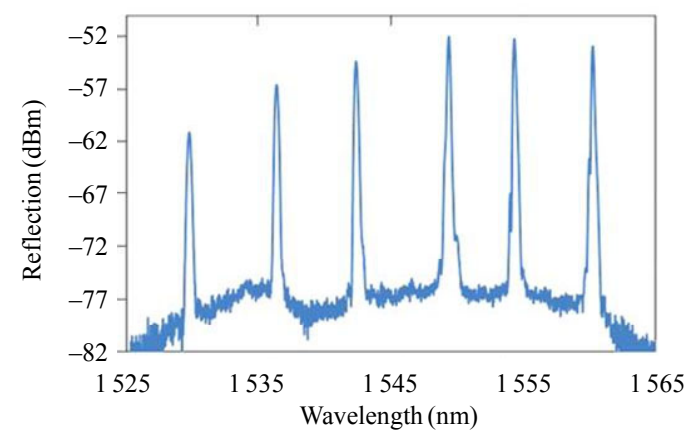

(b)

Fig. 4 Femtosecond laser holographic interferometry for fabricating FBGs: (a) schematics of experimental setup and (b) measured reflection spectrum of a fabricated FBG array [84].

\subsection{Femtosecond laser direct writing technology}

The femtosecond laser micromachining technology exhibits unique advantages of high accuracy and high quality with minimized thermal effect [11]. Refractive index modifications with a feature size of sub microns could be created inside the transparent material by tightly focusing the femtosecond laser beam via a high NA objective. Consequently, FBGs could be fabricated by using femtosecond laser direct writing technology. We established an experimental setup for inscribing FBGs by using this technology. As shown in Fig. 5(a), the setup employs a frequency-doubled regenerative amplified $\mathrm{Yb}: \mathrm{KGW} \quad\left[\mathrm{KGd}\left(\mathrm{WO}_{3}\right)\right]$ femtosecond laser (Pharos, Light-conversion) with a wavelength of $514 \mathrm{~nm}$, a pulse width of $290 \mathrm{fs}$, and a repetition rate of $200 \mathrm{kHz}$. A waveplate and Glan polarizer are used to adjust the optical power and polarization of laser beam. A dichroic mirror is used for reflecting the $514 \mathrm{~nm}$ femtosecond laser beam to 
the Zeiss oil-immersion objective $(63 \times, \mathrm{NA}=1.40)$, and is also used for transmitting the visible illumination beam to the charge-coupled device (CCD). The optical fiber is clamped by a pair of fiber holders mounted on a three-dimensional air-bearing translation stage (assembled by Aerotech ABL15010, ANT130LZS, and ANT130V-5). The top surface of the translation stage is tuned to be perpendicular to the incident beam. Note that no pre-removal of fiber coatings is required in this setup. During the FBG inscription process, the laser beam is fixed and the optical fiber is moved by the translation stage, and the top-view microscope images of fiber core is captured by the CCD for real-time positioning the laser beam focus inside the fiber core center. The femtosecond laser direct writing technology used for fabricating FBGs includes two different methods, i.e., point-by-point $(\mathrm{PbP})$ inscription method and line-by-line (LbL) scanning method. For the PbP inscription method, as shown in Fig. 5(b), the fiber is translated along its axis (i.e., the $x$ direction), and hence the focused laser pulses could induce periodical refractive index modulation on the fiber core. By adjusting the translation velocity $v$ or the repetition rate $f$ of the femtosecond laser pulses, the FBG period can be changed flexibly through $\Lambda=v / f$. For the LbL scanning method, as shown in Fig.5(c), the fiber is first translated along the $y$ axis (red line), and then translated along a diagonal line (blue dash line) via synchronous movements along the $x$ axis and $y$ axis. The shutter is closed during this step to prevent laser inscription. By repeating the same process, a Bragg grating with a grating pitch of $\Lambda$, a track length of $L$, and a pitch quantity of $N$, is formed by these lines inscribed in the fiber core. The top-view microscopic images of two FBGs inscribed with a $\mathrm{PbP}$ inscription method and an LbL scanning method are shown in Figs. 5(d) and 5(e), respectively. As the corresponding transmission and reflection spectra of these FBGs shown in Figs. 5(f) and $5(\mathrm{~g})$, both the PbP FBG and LbP FBG fabricated in our lab exhibit high reflectivity and low insertion loss.

In 2004, Martinez et al. [19] first reported the inscription of FBGs by using a NIR femtosecond laser PbP technology. Then, they achieved the FBG inscription through the fiber coating based on this technology, and the fabricated FBG exhibited excellent mechanical strength [87]. Since the refractive index modulation in cross-section of fiber induced by femtosecond laser $\mathrm{PbP}$ technology is not uniform, the transmission spectrum of FBG exhibits strong coupling to cladding modes [88, 89]. To solve this problem, we demonstrated the fabrication of FBGs in thin core fiber, which can effectively decrease the coupling to cladding mode [23]. In addition, the non-uniform modulation cross-section would raise the grating birefringence, and hence results in high PDL of FBG [90]. We reported a parallel-integrated fiber Bragg grating (PI-FBG) in SMF by using the femtosecond laser point-by-point technology [22]. By symmetrically arranging the PI-FBGs on the cross-section of the fiber core, we could achieve a PI-FBG with high reflectivity and low PDL. In recent years, the beam shaping technology has been demonstrated to improve the spectral quality of FBGs. Salter et al. [91] reported a specific adaptive optical arrangement to reduce the PDL of an FBG fabricated with femtosecond laser $\mathrm{PbP}$ technology. The aberration in the FBG fabrication process can be compensated by modifying the wave front with a spatial light modulator (SLM). Lu et al. [92] reported a modified $\mathrm{PbP}$ technology by adding a cylindrical lens to the fabrication setup, in which the cylindrical modulation was transformed into a planar modulation covering the fiber core. This plane-by-plane approach was proven an effective way for fabricating strong ( $25 \mathrm{~dB})$, low insertion loss $(<0.02 \mathrm{~dB})$, and complex grating structures in fiber. In addition, Varona et al. [93] used the slit 
beam technology to induce a wider and more circular plane on the fiber core by femtosecond laser. The FBG inscribed with this technology showed lower losses in transmission, as well as better reflectivity and full width half maximum (FWHM) than conventional $\mathrm{PbP}$ gratings. Furthermore, image recognition was introduced and used in the femtosecond $\mathrm{PbP}$ technology for auto-aligning the position of laser focus with the fiber core center, and hence could realize mass production of high-quality FBGs [94]. It is worth noted that this auto-alignment technology could also be used to fabricate long FBGs [95].
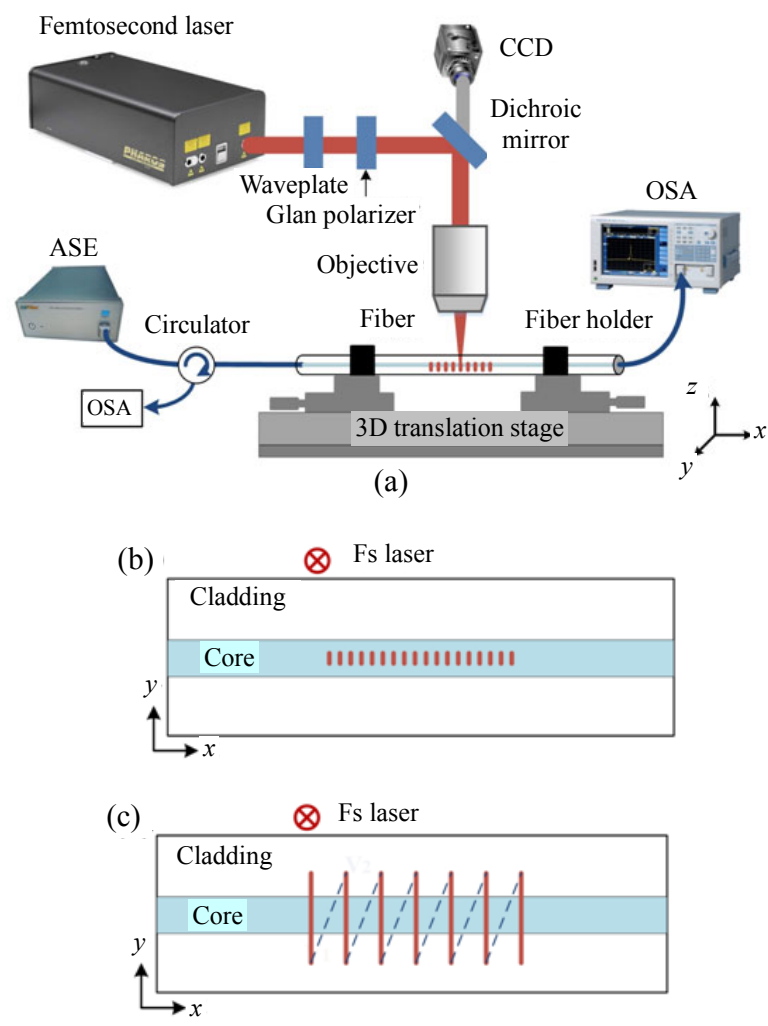

(d)

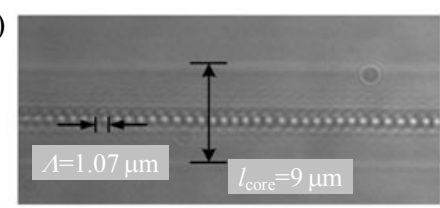

(e)

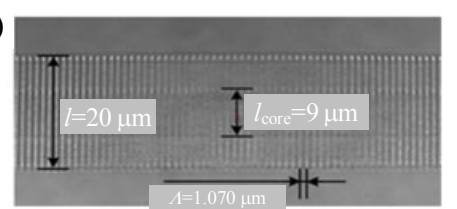

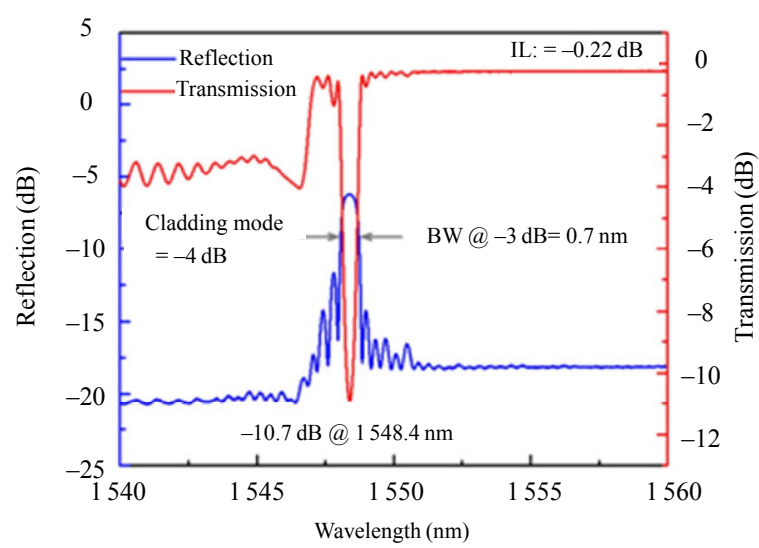

(f)

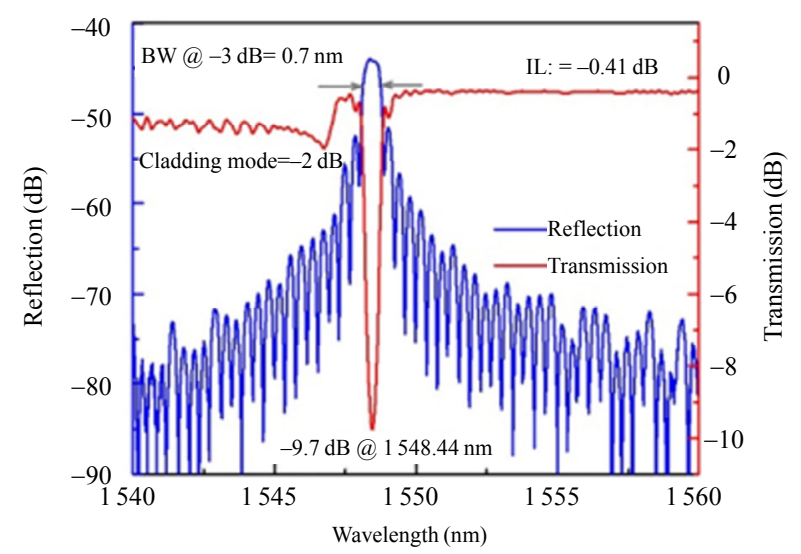

(g)

Fig. 5 Femtosecond laser direct writing technology for fabricating FBGs. Schematics of (a) the experimental setup, (b) the point-by-point $(\mathrm{PbP})$ inscription method, and (c) the line-by-line (LbL) scanning method. Top-view microscope images of two FBGs fabricated by using (d) PbP inscription method and (e) LbL scanning method, and the corresponding transmission and reflection spectra of the (f) PbP FBG and (g) LbL FBG.

In 2010, Zhou et al. [96] first reported a femtosecond laser LbL technology for inscribing high-quality FBGs. The fabricated LbL FBGs exhibited stronger Bragg resonance, lower insertion loss, and lower PDL than conventional PbP FBGs. Subsequently, in 2013, as shown in Fig. 6(a), Williams et al. [21] at Macquarie University proposed and demonstrated a piezo stage-based continuous scanning method for fabricating FBGs. As the transmission spectra shown in Fig.6(c), the FBG fabricated by using this method exhibits a high reflectivity $(-49 \mathrm{~dB})$ and low insertion loss $(\sim 0.1 \mathrm{~dB})$. In addition, some modified LbL inscription methods have also been reported and 
used for fabricating special FBGs. For example, Chah et al. [97] inscribed a highly birefringent FBG in conventional SMF by using a UV femtosecond laser LbL scanning technology. A high birefringence of $7.93 \times 10^{-4}$ was induced by these high-energy UV-laser-induced high-densification lines shifted from the center of fiber core. In 2019, Bharathan et al. [44] demonstrated single-pass (line by line), double-pass, and stacking (plane by plane) inscription techniques for directly writing FBGs in ZBLAN fibers with a high reflectivity $(\sim 90 \%)$ and low insertion loss $(<0.5 \mathrm{~dB})$.

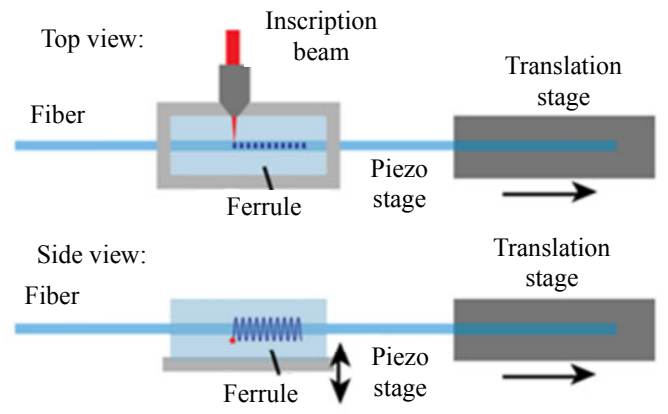

(a)

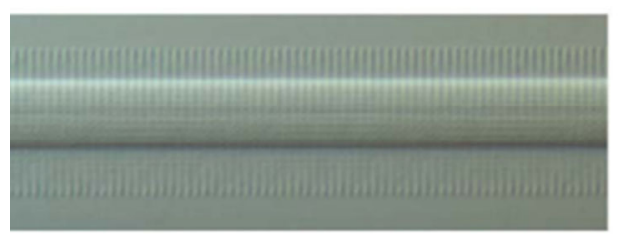

(b)

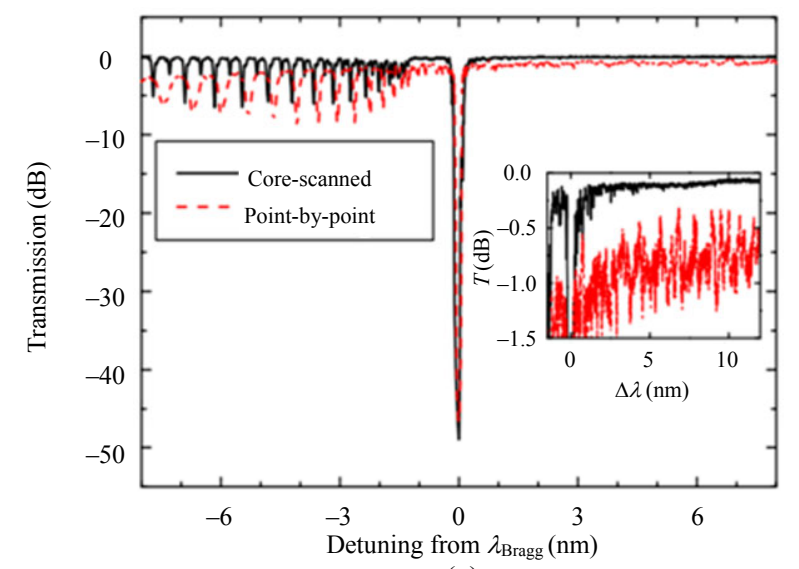

(c)

Fig. 6 Femtosecond laser core-scanning technology for fabricating high-quality FBGs: (a) schematics of experimental setup, (b) side-view microscope image of a core-scanned FBG, and (c) transmission spectra of a core-scanned FBG and a PbP grating with similar grating strength [21].

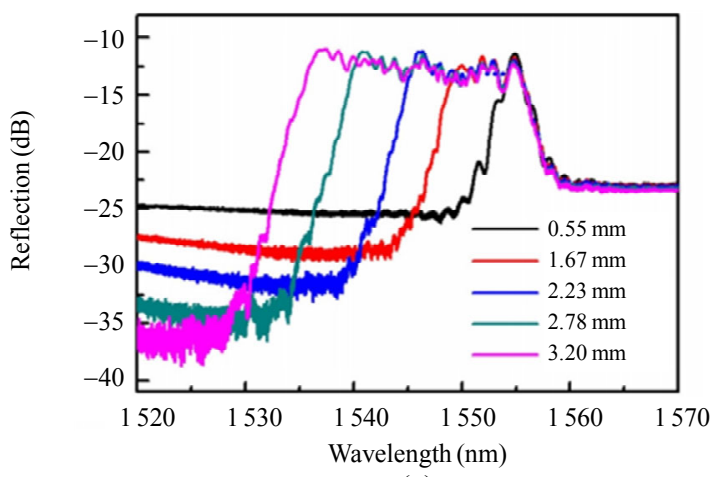

(a)

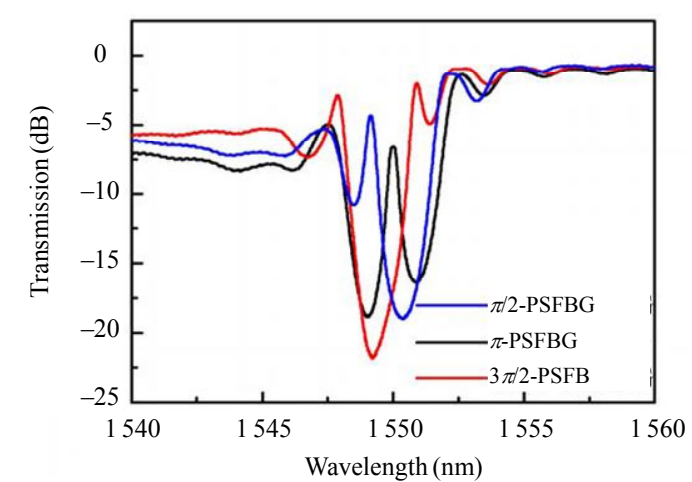

(b)

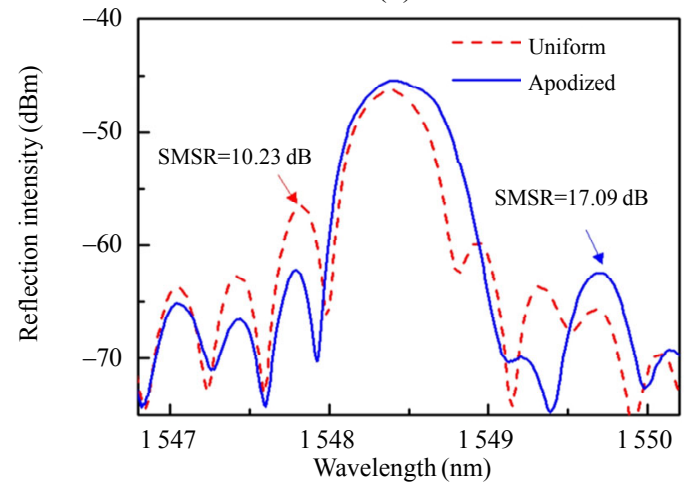

(c)

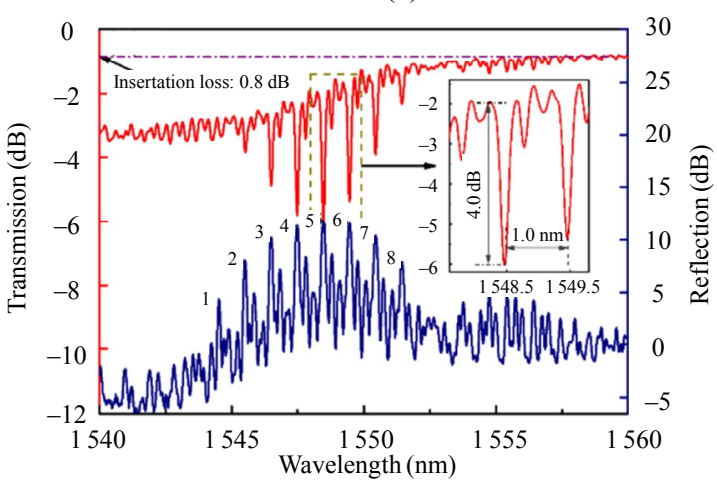

(d)

Fig. 7 Spectra of complex FBGs inscribed at Shenzhen University by using femtosecond laser direct writing technology. (a) chirped FBGs, (b) phase-shifted FBGs, (c) apodized FBGs inscribed by using LbL scanning technology [27], and (d) a sampled FBG fabricated by using an improved femtosecond laser PbP technology [26]. 
Furthermore, the femtosecond laser direct writing technology has demonstrated a remarkable flexibility for fabricating various types of FBGs, such as chirped FBGs, phase-shifted FBGs, apodized FBGs, or sampled FBGs. For example, in 2010, Marshall et al. reported complex FBGs fabricated by the PBP technology. By tailoring the position, phase, and amplitude of grating's refractive index modulations, they realized chirped gratings, sampled gratings, phase-shifted gratings [24] and apodized gratings [25]. As shown in Fig. 7, we also fabricated special FBGs with complex spectral shapes by using femtosecond laser direct writing technology. We successfully fabricated chirped FBGs, phase-shifted FBGs, and apodized FBGs in SMFs by using an LbL scanning technology, in which the line spacing, line position, and length of the inscribed tracks were arranged according to the design [27, 98]. Moreover, we also fabricated femtosecond laser PbP technology [26], as shown in Fig. 7(d). By periodic switching the highspeed shutter, the sampling parameters of the fiber grating can be accurately controlled.

\section{Femtosecond-laser-inscribed FBGs for high-temperature sensing applications}

Femtosecond laser-inscribed FBGs are suitable for high-temperature sensing in harsh environments, such as oil and gas industries, power stations, and aero engines. Various FBG-based high-temperature sensors have been reported in the past few decades. For example, FBGs inscribed by using the femtosecond laser $\mathrm{PbP}$ technology can withstand a high temperature of $900{ }^{\circ} \mathrm{C}$ [99]. The thermal stability of FBGs mainly depends on the refractive index modulation types induced by femtosecond laser with different pulse energies. Hence, the FBGs can be simply divided into type I FBG and type II FBG according to the different durable temperatures [100]. The type I grating occurs below the damage threshold of the medium. The index change is a result of highly nonlinear defect formation resulting from a multi-photon absorption process. The type II grating is a consequence of permanent damage to the medium after the process of ionization [60]. When the temperature is higher than $500{ }^{\circ} \mathrm{C}$, the type I grating will be erased. For example, NIR femtosecond laser-inscribed type I gratings can withstand temperatures of up to $500{ }^{\circ} \mathrm{C}$ [64]. However, type II gratings have much better thermal stability with no degradation at $1000^{\circ} \mathrm{C}$. In addition, the type II grating inscribed in pure-silica PCFs or single crystal sapphire fiber can also be created for sensing at a higher temperature of $1300^{\circ} \mathrm{C}$ [56] or an extremely high temperature of up to $1900^{\circ} \mathrm{C}$ [57].

\subsection{FBGs array for high-temperature sensing up to $700{ }^{\circ} \mathrm{C}$}

We reported on a high-quality FBG with a very low short wavelength loss inscribed in a small-core SMF by using the femtosecond laser PbP technology [23]. Moreover, a WDM FBGs array with low short wavelength loss was also fabricated in a small-core SMF, as shown in Figs. 8(a) and 8(b). And then, we investigated the thermal stability of an FBG array consisting of two FBGs annealed at $700^{\circ} \mathrm{C}$ for 195 hours. As shown in Fig. 8(c), the Bragg wavelength of FBG1 and FBG2 exhibits a 'blue' shift during the first 50 hours, and tends to be stable after annealing for 50 hours. This is resulted from the relaxation of internal stress in the FBG at the very beginning of the annealing process and the subsequent formation of an extremely stable grating structure after a long-term annealing process.

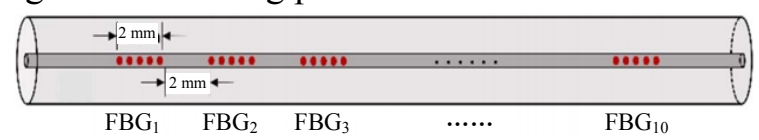

(a)

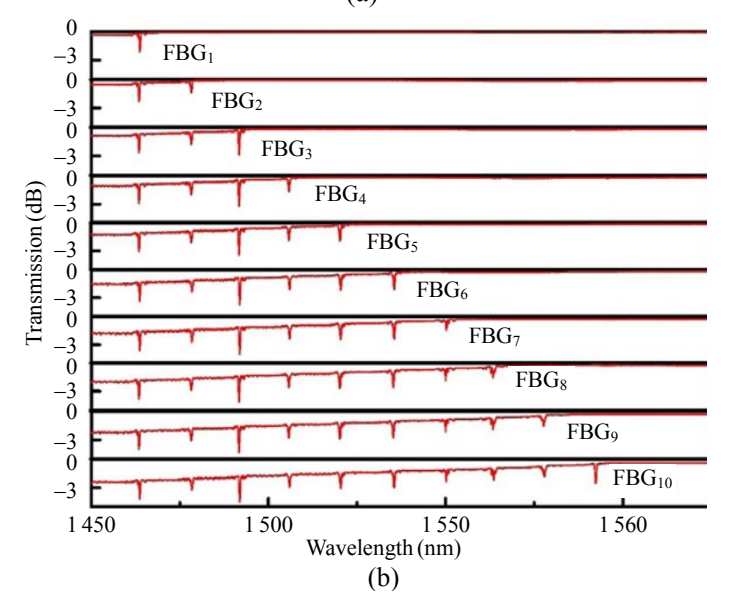




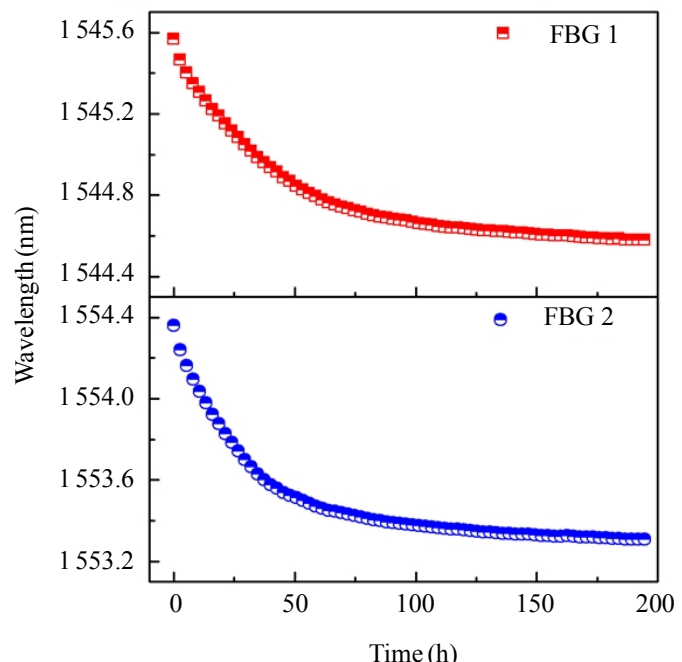

(c)

Fig. 8 Femtosecond laser-inscribed FBGs array for high-temperature sensing: (a) schematic diagram and (b) transmission spectra of the WDM-FBGs array [23]. (c) Long-term annealing study of a WDM-FBGs array consisting of FBG1 and FBG2.

\subsection{Negative-index FBGs for high-temperature sensing up to $1000{ }^{\circ} \mathrm{C}$}

The type II grating inscribed by femtosecond laser has excellent high temperature stability which can withstand temperatures of up to $1000{ }^{\circ} \mathrm{C}$. However, type II gratings always have poor spectral shapes and relatively larger insertion loss. Type IIA FBGs with negative-index change are typically reported in $\mathrm{H}_{2}$-free fibers by $\mathrm{UV}$ overexposure together with thermal regeneration. Such Type IIA FBGs can be developed for promising high temperature sensors. For example, Cook et al. [101] proposed a regenerative FBG fabricated by using near infrared femtosecond laser exposure and heat regeneration treatment in 2012, and such an FBG can also withstand a temperature of $1200{ }^{\circ} \mathrm{C}$. However, its regeneration efficiency is low, and the reflectivity of regenerated grating is less than $8 \%$. To solve this problem, we proposed a negativeindex FBG by use of a femtosecond laser overexposure and thermal regeneration in 2016 [102]. This negative-index FBG exhibits a high reflectivity of $99.22 \%$, an ultra-low insertion loss of $0.08 \mathrm{~dB}$, and an operating temperature of up to $1000{ }^{\circ} \mathrm{C}$. The fabrication process of the negative-

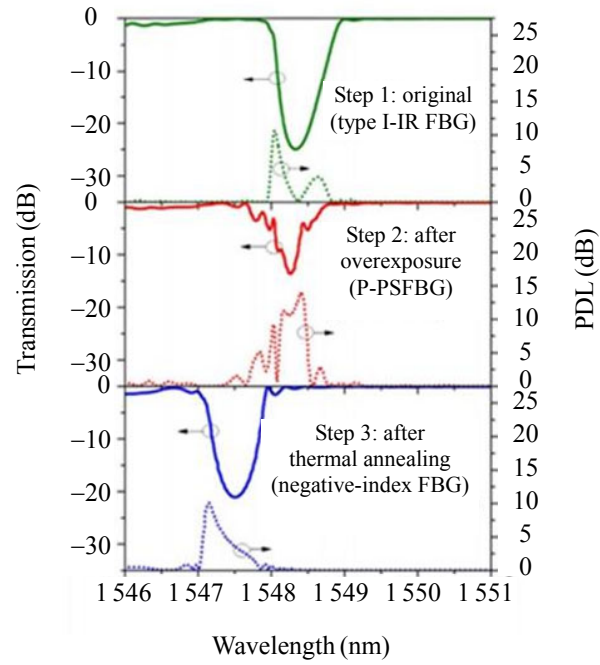

(a)

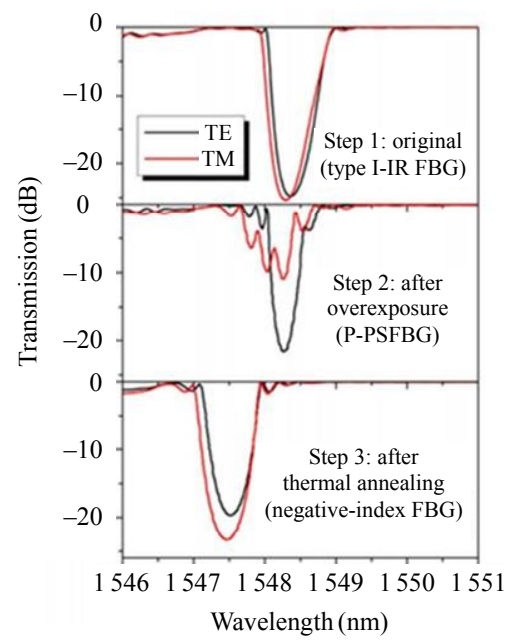

(b)

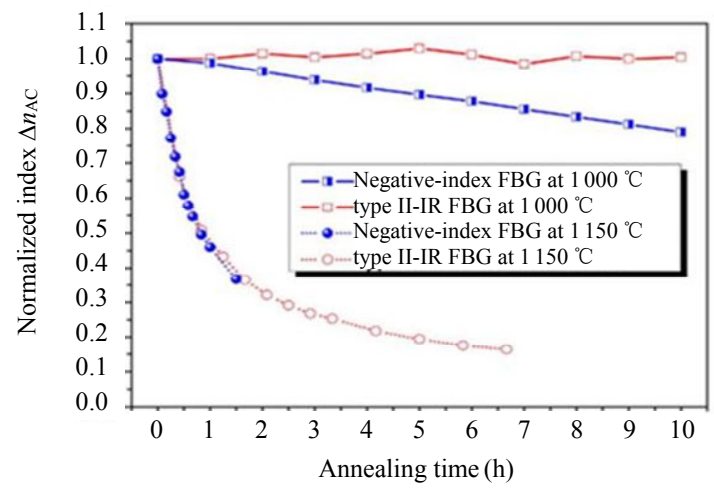

(c)

Fig. 9 High-temperature-resistant negative-index FBGs created by femtosecond laser overexposure and thermal regeneration: (a) transmission spectra and PDL, (b) transmission spectra of two orthogonal polarization modes (TE, TM) of FBGs fabricated from Step 1 to Step 3, and (c) evolution of normalized index modulation during long-term annealing [102].

index FBG involved three steps, as shown in Figs. 9(a) and 9(b). In Step 1, a positive-index type I-IR 
FBG was fabricated by a femtosecond laser inscription. In Step 2, the type I-IR FBG was further exposed to the femtosecond laser with the same pulse energy, to form a polarization-dependent phase-shifted FBG (P-PSFBG). In Step 3, the P-PSFBG was annealed at $800{ }^{\circ} \mathrm{C}$ for 12 hours, and a negative-index FBG was finally obtained. Note that the regeneration spectra of the TE and TM modes exhibit an observable 'blue' shift. This reveals the grating was formed by a negative-index modulation induced by the NIR femtosecond laser overexposure and thermal regeneration. Subsequently, we studied the thermal stabilities of the fabricated negative-index FBG and a conventional type II FBG. As shown in Fig. 9(c), neither of the two FBGs can withstand a high temperature of $1150^{\circ} \mathrm{C}$. Moreover, the type II FBG is quite stable at a high temperature of $1000{ }^{\circ} \mathrm{C}$, whereas the negative-index FBG degenerates gradually with an increase in annealing time. The high temperature stability of the thermally regenerated negative-index FBG is lower than that of a conventional type II-IR FBG, but much higher than that of a conventional type I-IR FBG.

\subsection{FBGs fabricated in pure-silica PCF for high-temperature sensing up to $1100{ }^{\circ} \mathrm{C}$}

The FBG fabricated in conventional SMF cannot withstand temperatures of up to $1000{ }^{\circ} \mathrm{C}$, since the fiber was shown to become extremely brittle at such high temperatures [103]. One promising approach to create FBGs operating at high temperatures of $\sim 1000{ }^{\circ} \mathrm{C}$ is to use undoped (e.g. pure fused silica) optical fibers, such as PCFs, in which the dopant diffusion at high temperatures could be avoided.

(a)

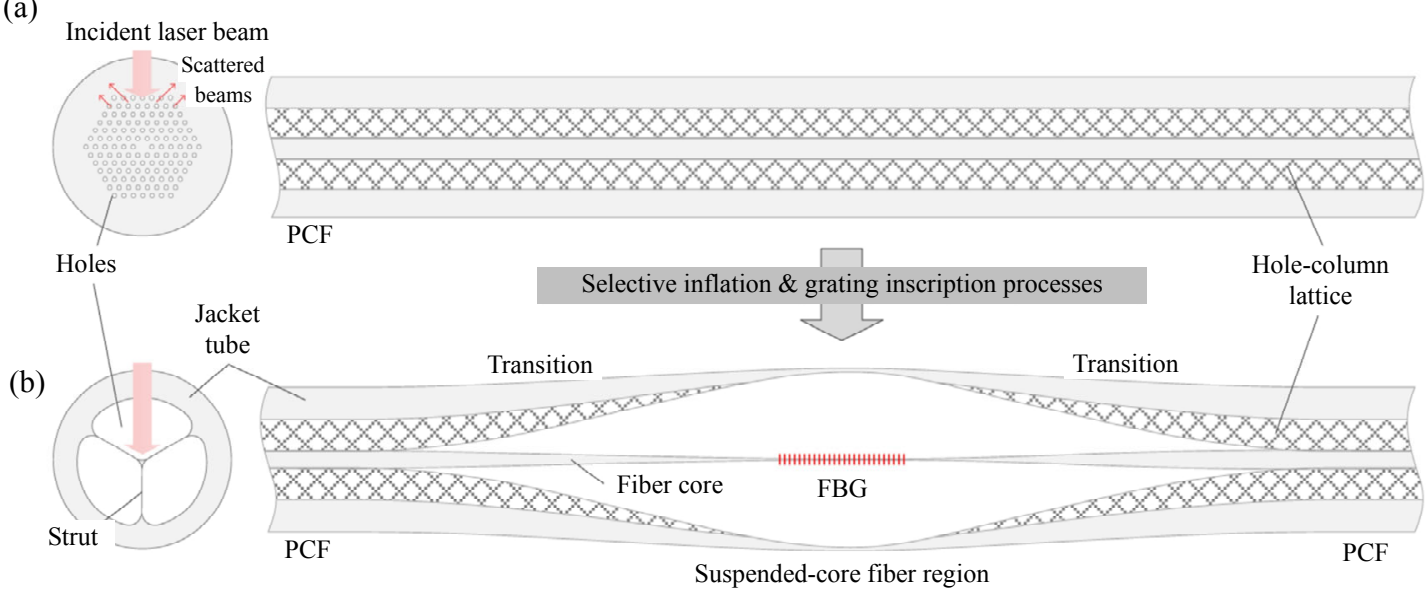

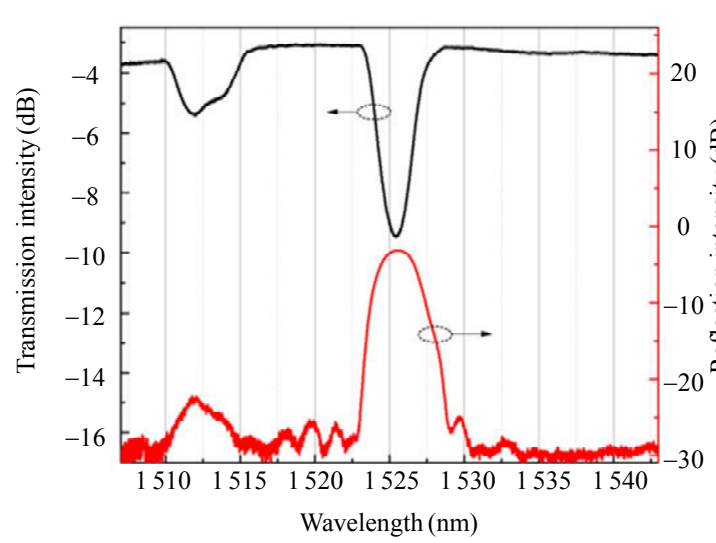

(c)

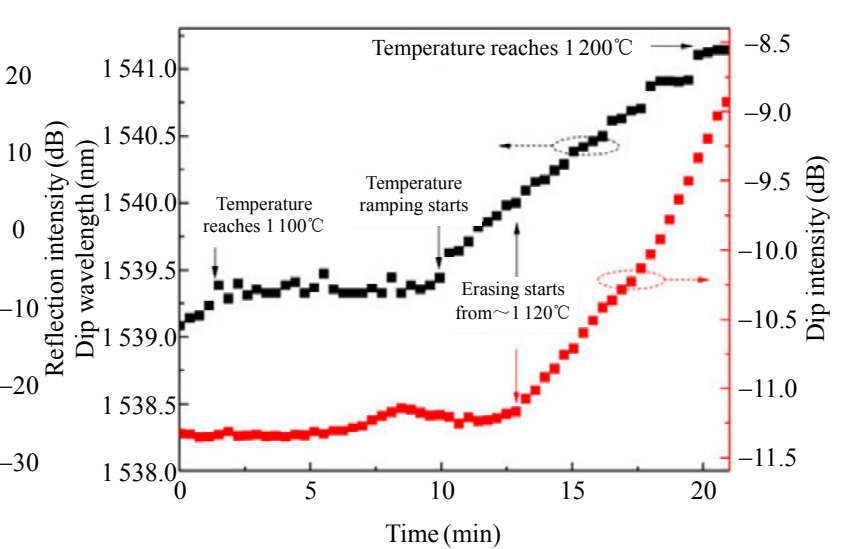

(d)

Fig. 10 Femtosecond laser-inscribed FBGs in pure-silica PCF for high-temperature sensing: (a) schematics of a commercial PCF, (b) the cross-section of a three-hole SCF region and Bragg grating inscription at the selectively inflated region [37], (c) transmission and reflection spectra of an FBG inscribed in an SCF, and (d) dip wavelength and intensity of FBG as functions of time in an annealing from $1100{ }^{\circ} \mathrm{C}$ to $1200{ }^{\circ} \mathrm{C}[104]$. 
There are many reports on the fabrication of FBGs in all-silica PCF for high-temperature applications. For example, Fu et al. [34] firstly reported on the fabrication of FBGs in PCF by using a UV femtosecond laser and found such FBGs would decay at a temperature of $500{ }^{\circ} \mathrm{C}$. The air holes layers in the PCF cladding lead to strong scattering and distortion of the incident femtosecond laser beam, and prevent the formation of a type II grating. To alleviate this problem, Warren-Smith et al. [56] fabricated type II FBGs in pure-silica suspendedcore PCFs. These FBGs can survive at a temperature of up to $1300{ }^{\circ} \mathrm{C}$. Moreover, we developed a method for FBG inscription in PCF by use of a phase mask and an infrared femtosecond laser at Shenzhen University. By selectively inflating a section of a commercial PCF, the PCF was transferred to a section of suspended-core fiber (SCF) with a simpler cladding structure, as show in Fig. 10(a). Hence, the inscription laser can laterally access to the core region with little loss [37]. As shown in Figs. 10(c) and 10(d), the FBG inscribed in SCF exhibits an excellent transmission and reflection spectra, as well as an enhanced thermal stability. The fabricated FBG in an SCF remains stable at a high temperature of $1000{ }^{\circ} \mathrm{C}$ for 10 hours and starts to decay at a higher temperature of $\sim 1120^{\circ} \mathrm{C}$ [104].

\subsection{FBGs inscribed in sapphire fiber for high-temperature sensing up to $1600{ }^{\circ} \mathrm{C}$}

The operating temperature of conventional silica-based FBG is limited by the glass transition temperature $\left(\sim 1330{ }^{\circ} \mathrm{C}\right)$ of silica. Single-crystal sapphire fiber has a very high melting temperature of $\sim 2045^{\circ} \mathrm{C}$, and hence it is a good candidate for high temperature sensing. In 2004, Mihailov et al. [46] first fabricated sapphire FBGs (SFBGs) by using a femtosecond laser phase mask technology, and demonstrated a high temperature sensing capability at $1500{ }^{\circ} \mathrm{C}$. By using a scanning beam phase mask method, the index modulation area could be enlarged, leading to a reflectivity enhancement in the fabricated SFBGs. Yu et al.
[105] reported on an SFBG with a reflectivity of $50 \%$ inscribed by using the same method, and these SFBGs can withstand high temperatures of $1690^{\circ} \mathrm{C}$. Moreover, Busch et al. [106] used the same method to fabricate high-performance SFBG sensors, featuring by a wide temperature operation range from $20^{\circ} \mathrm{C}$ to $1745^{\circ} \mathrm{C}$, a temperature measurement accuracy of $1{ }^{\circ} \mathrm{C}$, and good repeatability. In addition, they also studied the response of SFBGs at a temperature of up to $1900{ }^{\circ} \mathrm{C}$, which is the highest temperature achieved by using an SFBG so far [57].

Nevertheless, a WDM SFBGs array is difficult to be fabricated by using such a femtosecond laser phase mask technology since the wavelength of these SFBGs is fixed by the phase mask period. Elsmann et al. [47] successfully fabricated a WDM SFBGs array by using a $400 \mathrm{~nm}$ femtosecond laser holographic interferometry. Moreover, Wang et al. [48] proposed the inscription of FBGs in a single-crystal sapphire optical fiber via a femtosecond laser PbP technology. These methods provide a flexible way to fabricate WDM SFBGs array. However, the Talbot interferometer requires high stability and high accuracy, and the index modulation area inscribed by using PbP technology is small, leading to an extremely low reflectivity (i.e., $0.6 \%)$ of SFBG. In this case, we proposed the fabrication of SFBGs by using a femtosecond laser LbL scanning technique [49]. Figures 11(a) and 11(b) show the microscope images and reflection spectrum of the inscribed SFBG. This technology overcomes the defect of low reflectivity of SFBGs fabricated by $\mathrm{PbP}$ technology, and also has the advantages of flexibility in tuning the FBG period. Subsequently, an SFBG with a higher reflectivity of $6.3 \%$ can be obtained. A serial array consisting of five SFBGs at different wavelengths was successfully constructed, as shown in Fig. 11(c). Yu et al. [107] also realized the fabrication of SFBG with a reflectivity of $15 \%$ by using the same method. For further enhancing the reflectivity of SFBGs, we proposed the multi-layer grating structure to enlarge 
the index modulation area. An SFBG reflectivity of $34.1 \%$ can be achieved, which was much higher than that of a single-layer SFBG [108]. Moreover, both the single-layer and multi-layer SFBGs have

(a)
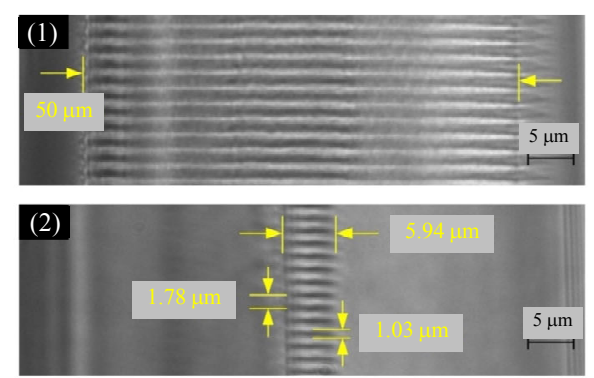

SFBG1 SFBG2 SFBG3 SFBG4 SFBG5

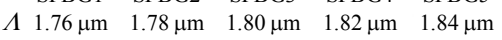

(c)
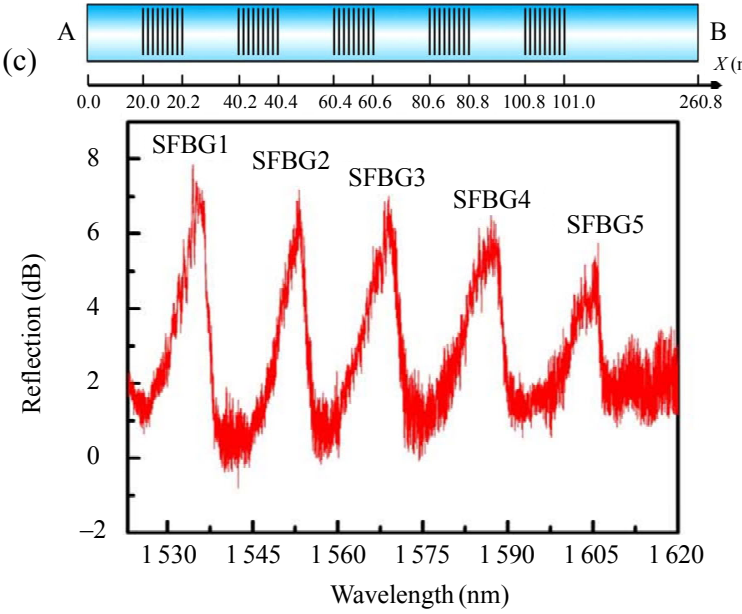

similar high-temperature characteristics. As shown in Fig. 11(d), the fabricated SFBG can withstand a high temperature of $1612{ }^{\circ} \mathrm{C}$ and exhibits a sensitivity of $45.2 \mathrm{pm} /{ }^{\circ} \mathrm{C}$ at $1612^{\circ} \mathrm{C}$.
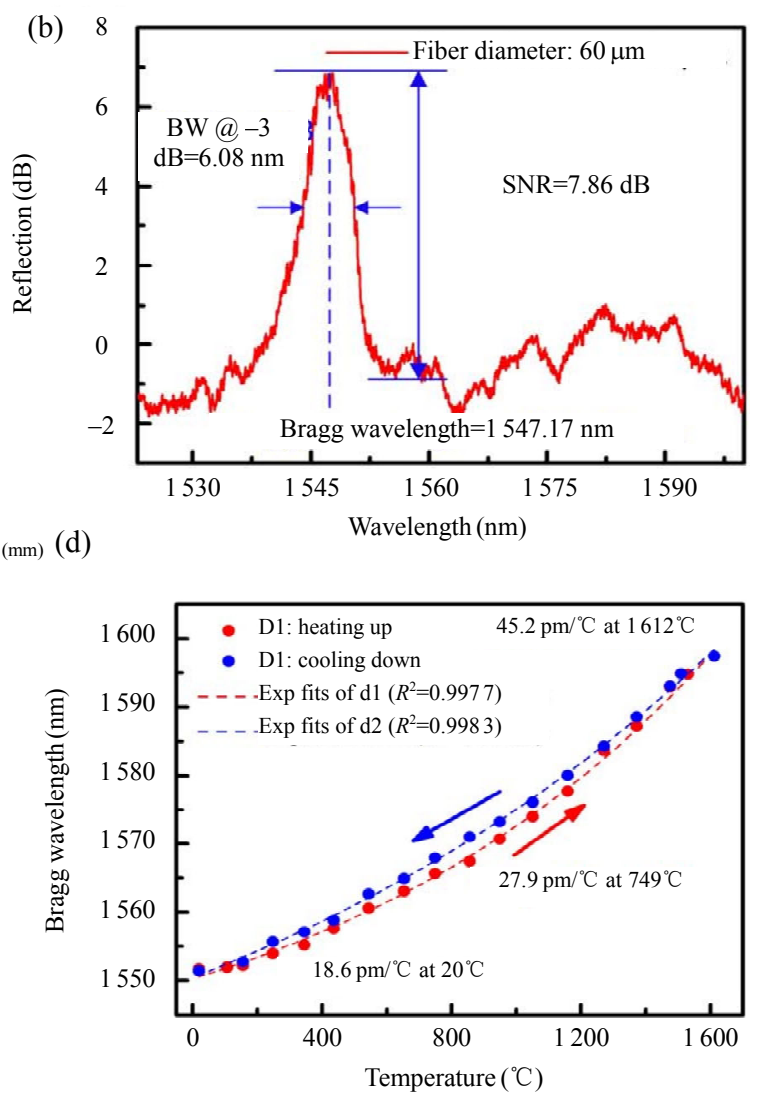

Fig. 11 FBGs array inscribed in sapphire fiber for high-temperature sensing: (a) microscope images of the inscribed SFBG. Reflection spectra of (b) an SFBG and (c) the SFBG array [49], and (d) Bragg wavelength of the SFBG as a function of the temperature in the case of temperature cycling from $20^{\circ} \mathrm{C}$ to $1612{ }^{\circ} \mathrm{C} \quad$ [108].

The SFBG exhibits a large bandwidth due to the multimode propagation at the wavelength of $1550 \mathrm{~nm}$. To date, three different methods, i.e., the tapered fiber coupling, wet-hot acid etching, and offset coupling have been proposed to reduce the SFBG bandwidth. Grobnic et al. [109] demonstrated a tapered SMF butt-coupled to the SFBG in which the SFBG bandwidth could be effectively reduced to $0.33 \mathrm{~nm}$. However, this method needs accurate alignment, which is not convenient for practical application. Yang et al. [110] proposed a method of wet-hot acid etching to reduce the diameter of the SFBG to $9.6 \mu \mathrm{m}$, in which the number of grating modes is effectively reduced and the quality of the reflective spectrum is further improved. However, it takes tens of hours in such a process. In addition, Elsmann et al. [111] inscribed FBGs in sapphire-derived all-glass optical fibers. Since the number of modes in the fabricated SFBGs decreased drastically, the SFBG bandwidth could be effectively reduced. However, this SFBG cannot operate at a temperature higher than $1000^{\circ} \mathrm{C}$. Hence, we proposed a method of offset coupling to suppress higher-order modes in the SFBG, and achieved a narrower $-3 \mathrm{~dB}$ bandwidth of $1.32 \mathrm{~nm}$ [108]. This method is simple and the fabricated SFBG can be used for high-temperature sensing up to $1612^{\circ} \mathrm{C}$.

Furthermore, SFBG can be employed as a dual strain/temperature sensor. Mihailov et al. [112] demonstrated a dual strain/temperature SFBG sensor 
operating at a high-temperature of $1288{ }^{\circ} \mathrm{C}$. By recording the blackbody radiation level and Bragg wavelength shifts, temperature and strain can be measured simultaneously. Habisreuther et al. [113] demonstrated an SFBG strain sensor with high temperature packaging, which could operate at a high temperature of $600{ }^{\circ} \mathrm{C}$ and had a strain resolution of $10 \mu \varepsilon$. Moreover, $\mathrm{Yu}$ et al. [105, 107] also achieved SFBG strain sensors with a sensitivity of $\sim 1.40 \mathrm{pm} / \mu \varepsilon$.

\section{Femtosecond-laser-inscribed FBGs for vector bending sensing applications}

Fiber-based vector bending sensor plays an important role in many applications, such as robotic arms and structure health monitoring (SHM). Conventional vector bending sensors based on long-period fiber gratings (LPGs) [114], off-axis FBGs [115], tilted FBGs [116], and eccentric FBGs [117], have been reported previously. In recent years, multicore fibers (MCFs) are developing rapidly. Hence, vector bending sensors based on FBGs inscribed on MCFs have also been reported [33, 58, 118]. These sensors could provide information on both bending magnitude and direction since the fiber cores distribute spatially in the MCF cross section.

\subsection{Parallel-integrated FBGs for bending sensing}

Conventional FBG inscription technology usually inscribes only one FBG in the cross section of the fiber core. As shown in Fig. 12(a), a femtosecond laser point-by-point technology was demonstrated to inscribe PI-FBGs in the fiber core cross section of an SMF [22]. We found that the grating parameters, such as reflectivity, reflection wavelength, PDL, and bandwidth, can be improved by changing the spatial distribution and grating numbers of the PI-FBGs. Moreover, as shown in Fig. 12(c), we used this technology to fabricate PI-FBGs including five FBGs with individual Bragg wavelengths in the reflection spectra and used these PI-FBGs for bending sensing. Owing to the small space (less than $2 \mu \mathrm{m}$ ) between each FBG and the fiber axis, the spatial distribution of the PI-FBGs does not significantly affect the bending property of the FBG, and hence the fabricated PI-FBG sample is insensitive to bending.

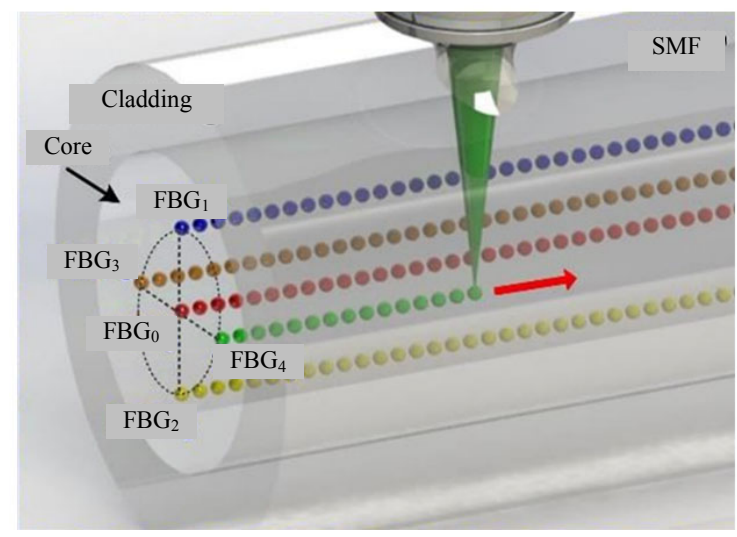

(a)

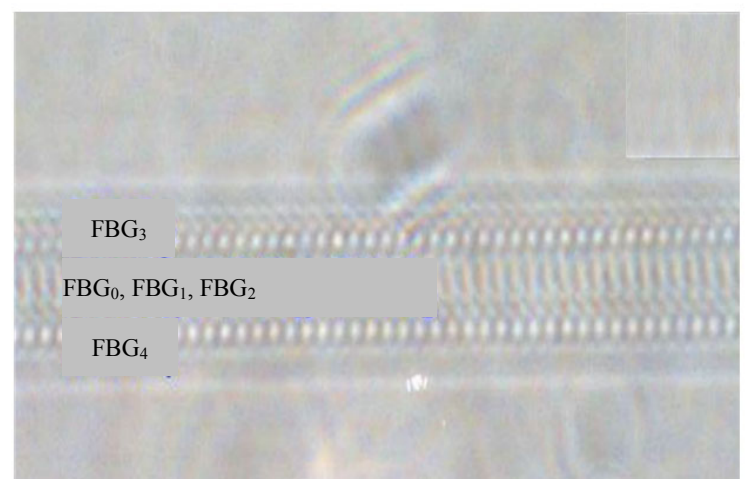

(b)

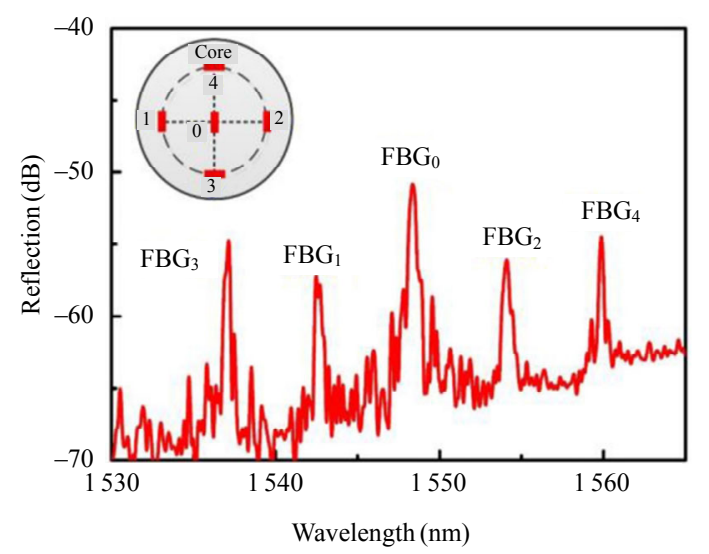

(c)

Fig. 12 Parallel-integrated FBGs (PI-FBGs) for directional bending sensing [22]: (a) schematics of the fabrication of PI-FBGs by using femtosecond laser direct writing technology, (b) side-view microscope image of the fabricated PI-FBG, and (c) corresponding reflection spectra of the PI-FBG including five FBGs with individual Bragg wavelengths. 


\subsection{Multicore FBGs for bending sensing}

At Shenzhen University, we also investigated the inscription of PI-FBGs in an MCF for bending sensing. We reported the inscription of FBG in a twin-core few-mode fiber (TC-FMF) for directional bend sensing [32]. The FBG was selectively inscribed in one core of the TC-FMF by using an $800 \mathrm{~nm}$ femtosecond laser through a phase mask. Since the fiber has few modes, three resonance peaks were observed in the reflection spectrum. As shown in Fig. 13(a), by changing the bend direction, we investigated the directional bend response of the TC-FMF FBG. It can be seen from Fig. 13(b) that the three resonant wavelengths exhibited a "red" shift in case $\theta$ changes from $0^{\circ}$ to $180^{\circ}$, and exhibited a "blue" shift in case $\theta$ changes from $180^{\circ}$ to $360^{\circ}$, which means the fabricated TC-FMF FBG has the capability of directional bend sensing.

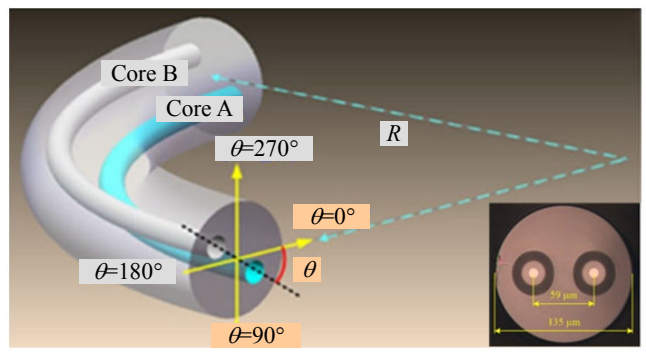

(a)

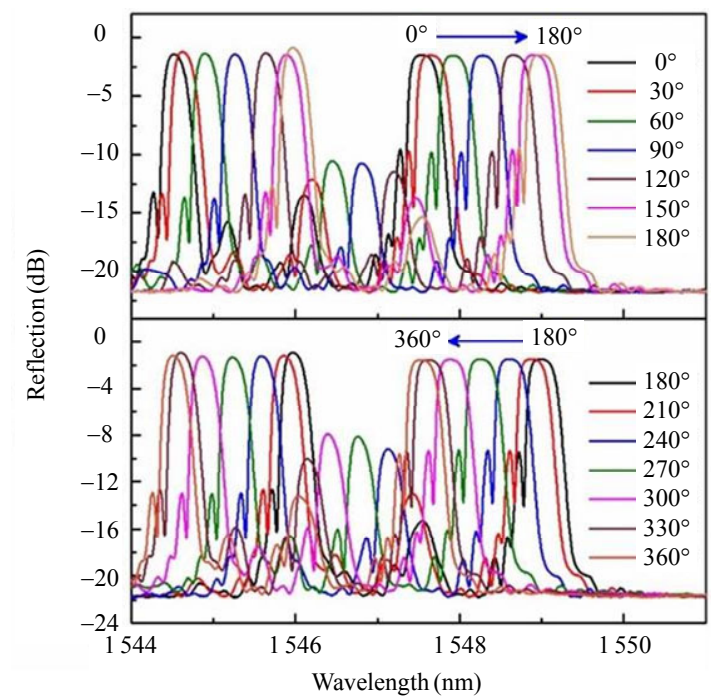

(b)

Fig. 13 Twin-core few-mode FBG (TC-FM FBG) for directional bending sensing [32]: (a) schematics of directional bending measurement for TC-FM FBG (inset: cross-sectional-view microscope image of the TC-FMF) and (b) reflection spectra evolution of the TC-FM FBG at different bending directions.
Directional bending sensors can only identify positive and negative directions. But in many engineering applications, two-dimensional bending (i.e., vector bending) sensors are in demand. Hence, we demonstrated a selective FBG inscription in four-core fiber (FCF) for two-dimensional vector bending sensing [119]. Two FBGs at different wavelengths were selectively inscribed into two different cores located at two orthogonal axes ( $y$-axis and $x$-axis). The wavelength responses of two FBGs can be utilized to synthesize a vector bending by monitoring the direction and amplitude when the FBG is under bending. Furthermore, we demonstrated a two-dimensional vector-bending sensor based on FBGs inscribed in a homogeneous seven-core fiber [86]. As shown in Fig. 14(a), the bending response of seven-core FBGs was investigated via a fan-in/out device and an exhibited

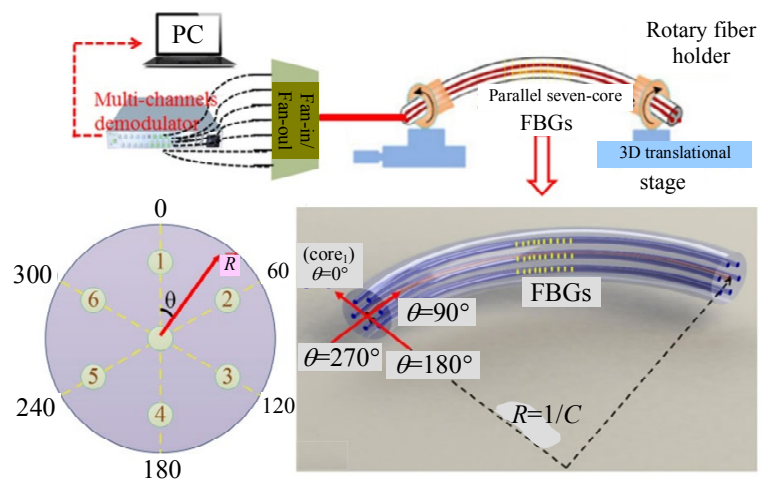

(a)

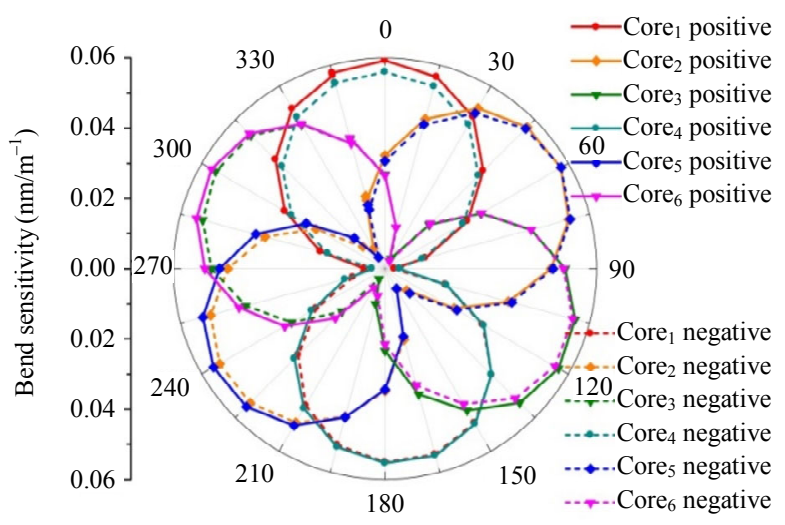

(b)

Fig. 14 Seven-core FBG for vector bending sensing [86]: (a) schematics of vector bending measurement for seven-core FBG and (b) bend sensitivities for the six outer-core FBGs plotted for various bending directions. 
strong bending-direction dependence, and multichannel demodulator. By changing bending curvature and orientation, the vector bending response of six outer-core FBGs was investigated at all $360^{\circ}$ directions with a step size of $15^{\circ}$. They displayed six perfect " 8 "-shaped patterns in a polar-coordinate systems shown in Fig. 14(b). Then, we used the measured Bragg wavelength shifts of any two off-diagonal outer-core FBGs to reconstruct orientation and amplitude of the vector bending.

\section{Conclusions}

We have reviewed the historical developments and recent advances in the fabrication technologies and sensing applications of various femtosecondlaser-inscribed FBGs. At first, we introduced the mechanism of femtosecond-laser-induced material changes, including three different types of refractive index modulation induced by femtosecond laser with different pulse energies. Subsequently, we introduced and studied three mainstream FBG fabrication technologies based on a femtosecond laser, i.e., femtosecond laser phase mask technology, femtosecond laser holographic interferometry, and femtosecond laser direct writing technology. The femtosecond laser phase mask technology is simple, stable, and efficient for fabricating FBGs with high reflectivity, low insertion loss, low cladding mode loss, and low PDL. However, it is difficult to use one phase mask to fabricate FBGs with different Bragg wavelengths. The femtosecond laser holographic interferometry can be used for fabricating WDM FBGs arrays due to its flexibility. However, it requires high precision, high stability, and high repeatability to establish such a Talbot interferometer due to the short coherence length (at tens of microns) in femtosecond laser. The femtosecond laser direct writing technology has the advantages of simplicity and flexibility. Various methods, such as beam shaping, image recognition, and core-scanning, have been proposed to improve the spectral quality of a femtosecond laser direct-written FBG, and the femtosecond laser direct writing technology can produce FBGs with similar spectral quality with the FBGs inscribed with a femtosecond laser phase mask technology. Moreover, the femtosecond laser direct writing technology has a remarkable flexibility for fabricating various types of FBGs. As a result, the femtosecond laser direct writing technology is the most promising way for fabricating FBGs at present, and hence we have carried out in-depth studies on this technology at Shenzhen University. And then, we introduced the progress in the applications of femtosecond-laser-inscribed for high-temperature sensing and vector bending sensing, respectively. We have successfully fabricated FBGs array in SMF, negative-index FBGs in SMF, FBGs in pure-silica PCF, and FBG arrays in single-crystal sapphire fibers, and used the fabricated FBGs for high-temperature sensing up to $700{ }^{\circ} \mathrm{C}, 1000^{\circ} \mathrm{C}, 1100{ }^{\circ} \mathrm{C}$, and $1600^{\circ} \mathrm{C}$, respectively. In addition, parallel integrated $\mathrm{FBGs}$ were also fabricated in MCF, and the MCF-FBGs can be used as vector bending sensors. In future, we will further develop the femtosecond laser direct writing technology to fabricate large-scale multiplexed ultra-weak FBGs arrays for high resolution, high precision, distributed high-temperature sensing, and three-dimensional vector bending sensing in harsh environments, and we will also explore the applications of femtosecond-laser-inscribed FBGs in many areas, such as aero engines, nuclear plants, power stations, smelting furnace, oil and gas industries, robotic arms, and medical instruments.

\section{Acknowledgment}

This work was supported by National Natural Science Foundation of China (NSFC) (Grant Nos. U1913212, 61875128, and 61635007); Department of Science and Technology of Guangdong Province (Grant Nos. 2019TQ05X113, 2019A1515011393, 2019B1515120042, and 2019A1515111114); Shenzhen Science and Technology Program (Grant Nos. RCYX20200714114538160 and JCYJ20180507182058432). 
Open Access This article is distributed under the terms of the Creative Commons Attribution 4.0 International License (http://creativecommons.org/licenses/by/4.0/), which permits unrestricted use, distribution, and reproduction in any medium, provided you give appropriate credit to the original author(s) and the source, provide a link to the Creative Commons license, and indicate if changes were made.

\section{References}

[1] T. Erdogan, "Fiber grating spectra," Journal of Lightwave Technology, 1997, 15(8): 1277-1294.

[2] K. O. Hill, Y. Fujii, D. C. Johnson, and B. S. Kawasaki, "Photosensitivity in optical fiber waveguides: application to reflection filter fabrication," Applied Physics Letters, 1978, 32(10): 647-649.

[3] G. Meltz, W. W. Morey, and W. H. Glenn, "Formation of Bragg gratings in optical fibers by a transverse holographic method," Optics Letters, 1989, 14(15): 823-825.

[4] P. J. Lemaire, R. M. Atkins, V. Mizrahi, and W. A. Reed, "High pressure $\mathrm{H}_{2}$ loading as a technique for achieving ultrahigh UV photosensitivity, and thermal sensitivity in $\mathrm{GeO}_{2}$ doped optical fibres," Electronics Letters, 1993, 29(13): 1191-1193.

[5] K. O. Hill, B. Malo, F. Bilodeau, D. C. Johnson, and J. Albert, "Bragg gratings fabricated in monomode photosensitive optical fiber by UV exposure through a phase mask," Applied Physics Letters, 1993, 62(10): 1035-1037.

[6] F. Ouellette, P. A. Krug, T. Stephens, G. Dhosi, and B. Eggleton, "Broadband and WDM dispersion compensation using chirped sampled fibre Bragg gratings," Electronics Letters, 1995, 31(11): 899-901.

[7] A. D. Kersey, M. A. Davis, H. J. Patrick, M. LeBlanc, K. P. Koo, and C. G. Askins "Fiber grating sensors," Journal of Lightwave Technology, 1997, 15(8): 1442-1463.

[8] J. Canning, "Fibre gratings and devices for sensors and lasers," Laser \& Photonics Reviews, 2008, 2(4): 275-289.

[9] J. Yao, "Optoelectronic oscillators for high speed and high-resolution optical sensing," Journal of Lightwave Technology, 2017, 35(16): 3489-3497.

[10] K. Itoh, W. Watanabe, S. Nolte, and C. B. Schaffer, "Ultrafast processes for bulk modification of transparent materials," MRS Bulletin, 2006, 31(8): 620-625.

[11] M. Beresna, M. GeceviIus, and P. G. Kazansky, "Ultrafast laser direct writing and nanostructuring in transparent materials," Advances in Optics \& Photonics, 2014, 6(3): 293-339.

[12] D. Pallarés-Aldeiturriaga, P. Roldán-Varona, L. Rodríguez-Cobo, and J. M. López-Higuera, "Optical fiber sensors by direct laser processing: a review," Sensors, 2020, 20(23): 6971.

[13] Y. Zhang, C. Lin, C. Liao, K. Yang, Z. Li, and Y. Wang, "Femtosecond laser-inscribed fiber interface Mach-Zehnder interferometer for temperature-insensitive refractive index measurement," Optics Letters, 2018, 43(18): 4421-4424.

[14] Y. Shimotsuma, P. G. Kazansky, J. Qiu, and K. Hirao, "Self-organized nanogratings in glass irradiated by ultrashort light pulses," Physical Review Letters, 2003, 91(24): 247405.

[15] F. Zhang, X. Xu, J. He, B. Du, and Y. Wang, "Highly sensitive temperature sensor based on a polymer-infiltrated Mach-Zehnder interferometer created in graded index fiber," Optics Letters, 2019 , 44(10): 2466-2469.

[16] K. Oi, F. Barnier, and M. Obara, "Fabrication of fiber Bragg grating by femtosecond laser interferometry," in 14th Annual Meeting of the IEEE Lasers and Electro-Optics Society, USA, 2001, pp. 776-777.

[17] A. Dragomir, D. N. Nikogosyan, K. A. Zagorulko, P. G. Kryukov, and E. M. Dianov, "Inscription of fiber Bragg gratings by ultraviolet femtosecond radiation," Optics Letters, 2003, 28(22): 2171-2173.

[18] S. J. Mihailov, C. W. Smelser, P. Lu, R. B. Walker, D. Grobnic, H. Ding, et al., "Fiber Bragg gratings made with a phase mask and 800-nm femtosecond radiation," Optics Letters, 2003, 28(12): 995-997.

[19] A. Martinez, M. Dubov, I. Khrushchev, and I. Bennion, "Direct writing of fibre Bragg gratings by femtosecond laser," Electronics Letters, 2004, 40(19): 1170-1172.

[20] D. Grobnic, C. W. Smelser, S. J. Mihailov, R. B. Walker, and P. Lu, "Fiber Bragg gratings with suppressed cladding modes made in SMF-28 with a femtosecond IR laser and a phase mask," IEEE Photonics Technology Letters, 2004, 16(8): 1864-1866.

[21] R. J. Williams, R. G. Krämer, S. Nolte, and M. J. Withford, "Femtosecond direct-writing of low-loss fiber Bragg gratings using a continuous core-scanning technique," Optics Letters, 2013, 38(11): 1918-1920.

[22] Y. Wang, Z. Li, S. Liu, C. Fu, Z. Li, Z. Zhang, et al., "Parallel-integrated fiber Bragg gratings inscribed by femtosecond laser point-by-point technology," Journal of Lightwave Technology, 2019, 37(10): 
2185-2193.

[23] X. Liu, Y. Wang, Z. Li, S. Liu, Yi. Wang, C. Fu, et al., "Low short-wavelength loss fiber Bragg gratings inscribed in a small-core fiber by femtosecond laser point-by-point technology," Optics Letters, 2019, 44(21): 5121-5124.

[24] G. D. Marshall, R. J. Williams, N. Jovanovic, M. J. Steel, and M. J. Withford, "Point-by-point written fiber-Bragg gratings and their application in complex grating designs," Optics Express, 2010, 18(19): 19844-19859.

[25] R. J. Williams, C. Voigtländer, G. D. Marshall, A. Tünnermann, S. Nolte, M. J. Steel, et al., "Point-by-point inscription of apodized fiber Bragg gratings," Optics Letters, 2011, 36(15): 2988-2990.

[26] C. Zhang, Y. Yang, C. Wang, C. Liao, and Y. Wang, "Femtosecond-laser-inscribed sampled fiber Bragg grating with ultrahigh thermal stability," Optics Express, 2016, 24(4): 3981-3988.

[27] K. Yang, C. Liao, S. Liu, J. He, J. Wang, and Y. Wang, "Optical fiber tag based on an encoded fiber Bragg grating fabricated by femtosecond laser," Journal of Lightwave Technology, 2019, 38(6): 1474-1479.

[28] B. Huang and X. Shu, "Ultra-compact strain- and temperature-insensitive torsion sensor based on a line-by-line inscribed phase-shifted FBG," Optics Express, 2016, 24(16): 17670-17679.

[29] J. Burgmeier, C. Waltermann, G. Flachenecker, and W. Schade, "Point-by-point inscription of phase-shifted fiber Bragg gratings with electro-optic amplitude modulated femtosecond laser pulses," Optics Letters, 2014, 39(3): 540-543.

[30] S. J. Mihailov, C. W. Smelser, D. Grobnic, R. B. Walker, P. Lu, H, Ding, et al., "Bragg gratings written in all- $\mathrm{SiO}_{2}$ and Ge-doped core fibers with 800 -nm femtosecond radiation and a phase mask," Journal of Lightwave Technology, 2004, 22(1): 94-100.

[31] C. M. Jewart, Q. Wang, J. Canning, D. Grobnic, S. J. Mihailov, and K. P. Chen, "Ultrafast femtosecond-laser-induced fiber Bragg gratings in air-hole microstructured fibers for high-temperature pressure sensing," Optics Letters, 2010, 35(9): 1443-1445.

[32] K. Yang, J. He, C. Liao, Y. Wang, S. Liu, K. Guo, et al., "Femtosecond laser inscription of fiber Bragg grating in twin-core few-mode fiber for directional bend sensing," Journal of Lightwave Technology, 2017, 35(21): 4670-4676.

[33] A. Wolf, A. Dostovalov, K. Bronnikov, and S. Babin, "Arrays of fiber Bragg gratings selectively inscribed in different cores of 7-core spun optical fiber by IR femtosecond laser pulses," Optics Express, 2019, 27(10): 13978-13990.

[34] L. B. Fu, G. D. Marshall, J. A. Bolger, P. Steinvurzel, E. C. Magi, M. J. Withford, et al., "Femtosecond laser writing Bragg gratings in pure silica photonic crystal fibres," Electronics Letters, 2005, 41(11): 638-640.

[35] S. J. Mihailov, D. Grobnic, H. Ding, C. W. Smelser, and J. Broeng, "Femtosecond IR laser fabrication of Bragg gratings in photonic crystal fibers and tapers," IEEE Photonics Technology Letters, 2006, 18(17): 1837-1839.

[36] T. Geernaert, K. Kalli, C. Koutsides, M. Komodromos, T. Nasilowski, W. Urbanczyk, et al., "Point-by-point fiber Bragg grating inscription in free-standing step-index and photonic crystal fibers using near-IR femtosecond laser," Optics Letters, 2010, 35(10): 1647-1649.

[37] C. Wang, J. He, J. Zhang, C. Liao, Y. Wang, W. Jin, et al., "Bragg gratings inscribed in selectively inflated photonic crystal fibers," Optics Express, 2017, 25(23): 28442-28450.

[38] S. J. Mihailov, D. Grobnic, C. W. Smelser, P. Lu, R. B. Walker, and H. Ding, "Bragg grating inscription in various optical fibers with femtosecond infrared lasers and a phase mask," Optical Materials Express, 2011, 1(4): 754-765.

[39] N. Jovanovic, A. Fuerbach, G. D. Marshall, M. J. Withford, and S. D. Jackson, "Stable high-power continuous-wave $\mathrm{Yb}^{3+}$-doped silica fiber laser utilizing a point-by-point inscribed fiber Bragg grating," Optics Letters, 2007, 32(11): 1486-1488.

[40] R. J. Williams, N. Jovanovic, G. D. Marshall, and M. J. Withford, "All-optical, actively Q-switched fiber laser," Optics Express, 2010, 18(8): 7714-7723.

[41] A. Fuerbach, G. Bharathan, and M. Ams, "Grating inscription into fluoride fibers: a review," IEEE Photonics Journal, 2019, 11(5): 1-11.

[42] D. Grobnic, S. J. Mihailov, and C. W. Smelser, "Femtosecond IR laser inscription of Bragg gratings in single-and multimode fluoride fibers," IEEE Photonics Technology Letters, 2006, 18(24): 2686-2688.

[43] D. Grobnic, S. J. Mihailov, C. W. Smelser, and R. Walker, "Bragg gratings made with ultrafast radiation in non-silica glasses; fluoride, phosphate, borosilicate and chalcogenide Bragg gratings," SPIE, Photonics North 2007, 6796: 67961K.

[44] G. Bharathan, T. T. Fernandez, M. Ams, R. I. Woodward, D. D. Hudson, and A. Fuerbach, "Optimized laser-written ZBLAN fiber Bragg gratings with high reflectivity and low loss," Optics Letters, 2019, 44(2): 423-426. 
[45] G. Bharathan, T. T. Fernandez, M. Ams, J. Carrée, S. Poulain, M. Poulain, et al., "Femtosecond laser direct-written fiber Bragg gratings with high reflectivity and low loss at wavelengths beyond 4 нm," Optics Letters, 2020, 45(15): 4316-4319.

[46] D. Grobnic, S. J. Mihailov, C. W. Smelser, and H. Ding, "Sapphire fiber Bragg grating sensor made using femtosecond laser radiation for ultrahigh temperature applications," IEEE Photonics Technology Letters, 2004, 16(11): 2505-2507.

[47] T. Elsmann, T. Habisreuther, A. Graf, M. Rothhardt, and H. Bartelt, "Inscription of first-order sapphire Bragg gratings using $400 \mathrm{~nm}$ femtosecond laser radiation," Optics Express, 2013, 21(4): 4591-4597.

[48] S. Yang, D. Hu, and A. B. Wang, "Point-by-point fabrication and characterization of sapphire fiber Bragg gratings," Optics Letters, 2017, 42(20): 4219-4222.

[49] X. Xu, J. He, C. Liao, K. Yang, K. Guo, C. Li, et al., "Sapphire fiber Bragg gratings inscribed with a femtosecond laser line-by-line scanning technique," Optics Letters, 2018, 43(19): 4562-4565.

[50] J. Thomas, C. Voigtlander, R. G. Becker, D. Richter, A. Tunnermann, and S. Nolte, "Femtosecond pulse written fiber gratings: a new avenue to integrated fiber technology," Laser \& Photonics Reviews, 2012, 6(6): 709-723.

[51] M. Bernier, R. Vallée, B. Morasse, C. Desrosiers, A. Saliminia, and Y. Sheng, "Ytterbium fiber laser based on first-order fiber Bragg gratings written with 400nm femtosecond pulses and a phase-mask," Optics Express, 2009, 17(21): 18887-18893.

[52] E. Wikszak, J. Thomas, J. Burghoff, B. Ortaç, J. Limpert, S. Nolte, et al., "Erbium fiber laser based on intracore femtosecond-written fiber Bragg grating," Optics Letters, 2006, 31(16): 2390-2392.

[53] M. Bernier, V. Michaud-Belleau, S. Levasseur, V. Fortin, J. Genest, and R. Vallée, "All-fiber DFB laser operating at $2.8 \mu \mathrm{m}$," Optics Letters, 2015, 40(1): 81-84.

[54] G. Bharathan, R. I. Woodward, M. Ams, D. D. Hudson, S. D. Jackson, and A. Fuerbach, "Direct inscription of Bragg gratings into coated fluoride fibers for widely tunable and robust mid-infrared lasers," Optics Express, 2017, 25(24): 30013-30019.

[55] D. Grobnic, C. W. Smelser, S. J. Mihailov, and R. B. Walker, "Long-term thermal stability tests at $1000^{\circ} \mathrm{C}$ of silica fibre Bragg gratings made with ultrafast laser radiation," Measurement Science and Technology, 2006, 17: 1009-1013.

[56] S. C. Warren-Smith, L. V. Nguyen, C. Lang, H. Ebendorff-Heidepriem, and T. M. Monro, "Temperature sensing up to $1300^{\circ} \mathrm{C}$ using suspended-core microstructured optical fibers," Optics Express, 2016, 24(4): 3714-3719.

[57] T. Habisreuther, T. Elsmann, Z. W. Pan, A. Graf, R. Willsch, and M. A. Schmidt, "Sapphire fiber Bragg gratings for high temperature and dynamic temperature diagnostics," Applied Thermal Engineering, 2015, 91: 860-865.

[58] K. Bronnikov, A. Wolf, S. Yakushin, A. Dostovalov, O. Egorova, S. Zhuravlev, et al., "Durable shape sensor based on FBG array inscribed in polyimide-coated multicore optical fiber," Optics Express, 2019, 27(26): 38421-38434.

[59] S. J. Mihailov, C. W. Smelser, and D. Grobnic, "Hydrogen loading for fiber grating writing with a femtosecond laser and a phase mask," Optics Letters, 2004, 29(18): 2127-2129.

[60] C. W. Smelser, S. J. Mihailov, and D. Grobnic, "Formation of Type I-IR and Type II-IR gratings with an ultrafast IR laser and a phase mask," Optics Express, 2005, 13(14): 5377-5386.

[61] E. Bricchi, B. G. Klappauf, and P. G. Kazansky, "Form birefringence and negative index change created by femtosecond direct writing in transparent materials," Optics Letters, 2004, 29(1): 119-121.

[62] J. He, Y. Wang, C. Liao, Q, Wang, K. Yang, B. Sun, et al., "Highly birefringent phase-shifted fiber Bragg gratings inscribed with femtosecond laser," Optics Letters, 2015, 40(9): 2008-2011.

[63] F. Yang, W. Jin, Y. Lin, C. Wang, H. Lut, and Y. Tan, "Hollow-core microstructured optical fiber gas sensors," Journal of Lightwave Technology, 2017, 35(16): 3413-3424.

[64] Y. Li, C. R. Liao, D. N. Wang, T. Sun, and K. T. V. Grattan, "Study of spectral and annealing properties of fiber Bragg gratings written in $\mathrm{H}_{2}$-free and $\mathrm{H}_{2}$-loaded fibers by use of femtosecond laser pulses," Optics Express, 2008, 16(26): 21239-21247.

[65] C. W. Smelser, S. J. Mihailov, D. Grobnic, P. Lu, R. B. Walker, H. Ding, et al., "Multiple-beam interference patterns in optical fiber generated with ultrafast pulses and a phase mask," Optics Letters, 2004, 29(13): 1458-1460.

[66] C. W. Smelser, D. Grobnic, and S. J. Mihailov, "Generation of pure two-beam interference grating structures in an optical fiber with a femtosecond infrared source and a phase mask," Optics Letters, 2004, 29(15): 1730-1732.

[67] S. J. Mihailov, D. Grobnic, and C. W. Smelser, "Efficient grating writing through fibre coating with femtosecond IR radiation and phase mask," Electronics Letters, 2007, 43(8): 442-443.

[68] M. Bernier, F. Trépanier, J. Carrier, and R. Vallée, 
"High mechanical strength fiber Bragg gratings made with infrared femtosecond pulses and a phase mask," Optics Letters, 2014, 39(12): 3646-3649.

[69] C. Hnatovsky, D. Grobnic, and S. J. Mihailov, "Through-the-coating femtosecond laser inscription of very short fiber Bragg gratings for acoustic and high temperature sensing applications," Optics Express, 2017, 25(21): 25435-25446.

[70] S. J. Mihailov, C. Hnatovsky, and D. Grobnic, "Novel type II Bragg grating structures in silica fibers using femtosecond lasers and phase Masks," Journal of Lightwave Technology, 2019, 37(11): 2549-2556.

[71] J. Thomas, C. Voigtländer, D. Schimpf, F. Stutzki, E. Wikszak, J. Limpert, et al., "Continuously chirped fiber Bragg gratings by femtosecond laser structuring," Optics Letters, 2008, 33(14): 1560-1562.

[72] C. Voigtländer, J. Thomas, E. Wikszak, P. Dannberg, S. Nolte, and A. Tünnermann, "Chirped fiber Bragg gratings written with ultrashort pulses and a tunable phase mask," Optics Letters, 2009, 34(12): 1888-1890.

[73] M. Bernier, Y. Sheng, and R. Vallée, "Ultrabroadband fiber Bragg gratings written with a highly chirped phase mask and Infrared femtosecond pulses," Optics Express, 2009, 17(5): 3285-3290.

[74] D. Grobnic, S. J. Mihailov, and C. W. Smelser, "Localized high birefringence induced in SMF-28 fiber by femtosecond IR laser exposure of the cladding," Journal of Lightwave Technology, 2007, 25(8): 1996-2001.

[75] Q. Rong, X. Qiao, T. Guo, W. Bao, D. Su, and H. Yang, "Orientation-dependent fiber-optic accelerometer based on grating inscription over fiber cladding," Optics Letter, 2014, 39(23): 6616-6619.

[76] L. Talbot, P. Paradis, and M. Bernier, “All-fiber laser pump reflector based on a femtosecond-written inner cladding Bragg grating," Optics Letter, 2019, 44(20): 5033-5036.

[77] N. Abdukerim, D. C. Hnatovsky, and S. J. Mihailov, "High-temperature stable fiber Bragg gratings with ultra-strong cladding modes written using the phase mask technique and an infrared femtosecond laser," Optics Letter, 2020, 45(2): 443-446.

[78] M. Becker, J. Bergmann, S. Brückner, M. Franke, E. Lindner, M. W. Rothhardt, et al., "Fiber Bragg grating inscription combining DUV sub-picosecond laser pulses and two-beam interferometry," Optics Express, 2008, 16(23): 19169-19178.

[79] M. Becker, S. Brückner, M. Leich, E. Lindner, M. Rothhardt, S. Unger, et al., "Towards a monolithic fiber laser with deep UV femtosecond-induced fiber
Bragg gratings," Optics Communications, 2011, 284(24): 5770-5773.

[80] J. Fiebrandt, E. Lindner, S. Brückner, M. Becker, A. Schwuchow, M. Rothhardt, et al., "Growth characterization of fiber Bragg gratings inscribed in different rare-earth-doped fibers by UV and VIS femtosecond laser pulses," Optics Communications, 2012, 285(24): 5157-5162.

[81] M. Leich, J. Fiebrandt, S. Jetschke, M. Rothhardt, and M. Jäger, "In situ FBG inscription during fiber laser operation," Optics Letters, 2013, 38(5): 676-678.

[82] Y. Wang, H. Bartelt, M. Becker, S. Brueckner, J. Bergmann, J. Kobelke, et al., "Fiber Bragg grating inscription in pure-silica and Ge-doped photonic crystal fibers," Applied Optics, 2009, 48(11): 1963-1968.

[83] M. Becker, L. Fernandes, M. Rothhardt, S. Brückner, K. Schuster, J. Kobelke, et al., "Inscription of fiber Bragg grating arrays in pure silica suspended core fibers," IEEE Photonics Technology Letters, 2009, 21(19): 1453-1455.

[84] A. Saliminia and R. Vallée, "Fiber Bragg grating inscription based on optical filamentation of UV femtosecond laser pulses," Optics Communications, 2014, 324(15): 245-251.

[85] Z. Zhang, B. Xu, J. He, M. Hou, W. Bao, and Y. Wang, "High-efficiency inscription of fiber Bragg grating array with high-energy nanosecond-pulsed laser Talbot interferometer," Sensors, 2020, 20(15): 4307.

[86] M. Hou, K. Yang, J. He, X. Xu, S. Ju, K. Guo, et al., "Two-dimensional vector bending sensor based on seven-core fiber Bragg gratings," Optics Express, 2018, 26(18): 23770-23781.

[87] A. Martinez, I. Khrushchev, and I. Bennion, "Direct inscription of Bragg gratings in coated fibers by an infrared femtosecond laser," Optics Letters, 2006, 31(11): 1603-1605.

[88] J. Thomas, N. Jovanovic, R. G. Becker, G. D. Marshall, M. J. Withford, A. Tünnermann, et al., "Cladding mode coupling in highly localized fiber Bragg gratings: modal properties and transmission spectra," Optics Express, 2011, 19(1): 325-341.

[89] J. Thomas, N. Jovanovic, R. G. Becker, G. D. Marshall, M. J. Withford, A. Tünnermann, et al., "Cladding mode coupling in highly localized fiber Bragg gratings II: complete vectorial analysis," Optics Express, 2012, 20(19): 21434-21449.

[90] C. Caucheteur, T. Guo, and J. Albert, "Polarization-assisted fiber Bragg grating sensors: tutorial and review," Journal of Lightwave Technology, 2017, 35(16): 3311-3322. 
[91] P. S. Salter, M. J. Woolley, S. M. Morris, M. J. Booth, and J. A. J. Fells, "Femtosecond fiber Bragg grating fabrication with adaptive optics aberration compensation," Optics Letters, 2018, 43(24): 5993-5996.

[92] P. Lu, S. J. Mihailov, H. Ding, D. Grobnic, R. B. Walker, and D. Coulas, "Plane-by-plane inscription of grating structures in optical fibers," Journal of Lightwave Technology, 2017, 36(4): 926-931.

[93] P. Roldán-Varona, D. Pallarés-Aldeiturriaga, L. Rodríguez-Cobo, and J. M. López-Higuera, "Slit beam shaping technique for femtosecond laser inscription of enhanced plane-by-plane FBGs," Journal of Lightwave Technology, 2020, 38(16): 4526-4532.

[94] A. V. Dostovalov, A. A. Wolf, A. V. Parygin, V. E. Zyubin, and S. A. Babin, "Femtosecond point-by-point inscription of Bragg gratings by drawing a coated fiber through ferrule," Optics Express, 2016, 24(15): 16232-16237.

[95] Y. Yu, J. Shi, F. Han, W. Sun, and X. Feng, "High-precision fiber Bragg gratings inscription by infrared femtosecond laser direct-writing method assisted with image recognition," Optics Express, 2020, 28(6): 8937-8948.

[96] K. Zhou, M. Dubov, C. Mou, L. Zhang, V. K. Mezentsev, and I. Bennion, "Line-by-Line fiber Bragg grating made by femtosecond laser," IEEE Photonics Technology Letters, 2010, 22(16): 1190-1192.

[97] K. Chah, D. Kinet, M. Wuilpart, P. Mégret, and C. Caucheteur, "Femtosecond-laser-induced highly birefringent Bragg gratings in standard optical fiber," Optics Letters, 2013, 38(4): 594-596.

[98] J. Luo, S, Liu, Y. Zhao, Y. Chen, K. Yang, K. Guo, et al., "Phase-shifted fiber Bragg grating modulated by a hollow cavity for measuring gas pressure," Optics Letters, 2020, 45(2): 507-510.

[99] A. Martinez, I. Y. Khrushchev, and I. Bennion, "Thermal properties of fibre Bragg gratings inscribed point-by-point by infrared femtosecond laser," Eletronics Letters, 2005, 41(4): 6-7.

[100] C. Liao, Y. Li, D. N. Wang, T. Sun, and K. T. V. Grattan, "Morphology and thermal stability of fiber Bragg gratings for sensor applications written in $\mathrm{H}_{2}$-free and $\mathrm{H}_{2}$-loaded fibers by femtosecond laser," IEEE Sensors Journal, 2010, 10(11): $1675-1681$.

[101] K. Cook, L. Shao, and J. Canning, "Regeneration and helium: regenerating Bragg gratings in helium-loaded germanosilicate optical fibre," Optical Materials Express, 2012, 2(12): 1733-1742.
[102] J. He, Y. Wang, C. Liao, C. Wang, S. Liu, K. Yang, et al., "Negative-index gratings formed by femtosecond laser overexposure and thermal regeneration," Scientific Report, 2016, 6: 23379.

[103] C. R. Liao and D. N. Wang, "Review of femtosecond laser fabricated fiber Bragg gratings for high temperature sensing," Photonic Sensors, 2013, 3: 97-101.

[104] C. Wang, J. Zhang, C. Zhang, J. He, Y. Lin, W. Jin, et al., "Bragg gratings in suspended-core photonic microcells for high-temperature applications," Journal of Lightwave Technology, 2019, 36(14): 2920-2924.

[105] C. Chen, X. Zhang, Y. Yu, W. Wei, Q. Guo, L. Qin, et al., "Femtosecond laser-inscribed high-order Bragg gratings in large-diameter sapphire fibers for high-temperature and strain sensing," Journal of Lightwave Technology, 2018, 36(16): 3302-3308.

[106] M. Busch, W. Ecke, I. Latka, D. Fischer, R. Willsch, and H. Bartelt, "Inscription and characterization of Bragg gratings in single-crystal sapphire optical fibers for high-temperature sensor applications," Measurement Science and Technology, 2009, 20: 115301.

[107] Q. Guo, Y. Yu, Z. Zheng, C. Chen, P. Wang, Z. Tian, et al., "Femtosecond laser inscribed sapphire fiber Bragg grating for high temperature and strain sensing," IEEE Transactions on Nanotechnology, 2019, 18: 208-211.

[108] X. Xu, J. He, C. Liao, and Y. Wang, "Multi-layer, offset-coupled sapphire fiber Bragg gratings for high-temperature measurements," Optics Letters, 2019, 44(17): 4211-4214.

[109] D. Grobnic, S. J. Mihailov, H. Ding, F. Bilodeau, and C. W. Smelser, "Single and low order mode interrogation of a multimode sapphire fiber Bragg grating sensor with tapered fibers," Measurement Science and Technology, 2006, 17: 980-984.

[110] S. Yang, H. Daniel, P. Gary, and A. Wang, "Fiber Bragg grating fabricated in micro-single-crystal sapphire fiber," Optics Letters, 2018, 43(1): 62-65.

[111] T. Elsmann, A. Lorenz, N. S. Yazd, T. Habisreuther, J. Dellith, and A. Schwuchow, "High temperature sensing with fiber Bragg gratings in sapphire-derived all-glass optical fibers," Optics Express, 2014, 22(22): 26825-26833.

[112] S. J. Mihailov, D. Grobnic, and C. W. Smelser, "High-temperature multiparameter sensor based on sapphire fiber Bragg gratings," Optics Letters, 2010, 35(16): 2810-2812.

[113] T. Habisreuther, T. Elsmann, A. Graf, and M. A. Schmidt, "High-temperature strain sensing using sapphire fibers with inscribed first-order Bragg 
gratings," IEEE Photonics Journal, 2016, 8(3): 6802608.

[114] L. Jin, W. Jin, and J. Ju, "Directional bend sensing with a $\mathrm{CO}_{2}$-laser-inscribed long period grating in a photonic crystal fiber," Journal of Lightwave Technology, 2009, 27(21): 4884-4891.

[115] D. Feng, X. Qiao, and J. Albert, "Off-axis ultraviolet-written fiber Bragg gratings for directional bending measurements," Optics Letters, 2016, 41(6): 1201-1204.

[116] L. Shao, L. Xiong, C. Chen, A. Laronche, and J. Albert, "Directional bend sensor based on re-grown tilted fiber Bragg grating," Journal of Lightwave Technology, 2010, 28(18): 2681-2687.
[117] W. Bao, Q. Rong, F. Chen, and X. Qiao, "All-fiber $3 \mathrm{D}$ vector displacement (bending) sensor based on an eccentric FBG," Optics Express, 2018, 26(7): 8619-8627.

[118] D. Zheng, J. Madrigal, H. Chen, D. Barrera, and S. Sales, "Multicore fiber-Bragg-grating-based directional curvature sensor interrogated by a broadband source with a sinusoidal spectrum," Optics Letters, 2017, 42(18): 3710-3713.

[119] W. Bao, N. Sahoo, Z. Sun, C. Wang, S. Liu, Y. Wang, et al., "Selective fiber Bragg grating inscription in four-core fiber for two-dimension vector bending sensing," Optics Express, 2020, 28(18): 26461-26469. 